\title{
LOCATING PLANETESIMAL BELTS IN THE MULTIPLE-PLANET SYSTEMS HD 128311, HD 202206, HD 82943, AND HR 8799
}

\author{
Amaya Moro-Martín ${ }^{1,2}$, Renu Malhotra ${ }^{3}$, Geoffrey Bryden ${ }^{4}$, George H. Rieke ${ }^{5}$, Kate Y. L. Su ${ }^{5}$, \\ Charles A. Beichman ${ }^{6}$, and Samantha M. Lawler ${ }^{7}$ \\ ${ }^{1}$ Department of Astrophysics, Center for Astrobiology (CSIC-INTA), Ctra. de Ajalvir, km 4, Torrejón de Ardoz, 28850, Madrid, Spain \\ ${ }^{2}$ Department of Astrophysical Sciences, Princeton University, Peyton Hall, Ivy Lane, Princeton, NJ 08544, USA \\ ${ }^{3}$ Department of Planetary Sciences, University of Arizona, 1629 E. University Boulevard, Tucson, AZ 85721, USA \\ ${ }^{4}$ Jet Propulsion Laboratory, 4800 Oak Grove Drive, Pasadena, CA 91109, USA \\ ${ }^{5}$ Steward Observatory, University of Arizona, 933 North Cherry Ave., Tucson, AZ 85721, USA \\ ${ }^{6}$ NASA Exoplanet Science Institute, California Institute of Technology, Pasadena, CA 91125, USA \\ ${ }^{7}$ Department of Physics and Astronomy, University of British Columbia, 6224 Agricultural Road, Vancouver, BC V6T 1Z1, Canada \\ Received 2010 April 5; accepted 2010 May 14; published 2010 June 23
}

\begin{abstract}
In addition to the Sun, six other stars are known to harbor multiple planets and debris disks: HD 69830, HD 38529, HD 128311, HD 202206, HD 82943, and HR 8799. In this paper, we set constraints on the location of the dustproducing planetesimals around the latter four systems. We use a radiative transfer model to analyze the spectral energy distributions of the dust disks (including two new Spitzer IRS spectra presented in this paper), and a dynamical model to assess the long-term stability of the planetesimals' orbits. As members of a small group of stars that show evidence of harboring a multiple planets and planetesimals, their study can help us learn about the diversity of planetary systems.
\end{abstract}

Key words: circumstellar matter - infrared: stars - Kuiper Belt: general - planetary systems - stars: individual (HD 128311, HD 202206, HR 8799, HD 82943)

\section{INTRODUCTION}

Surveys with the Spitzer Space Telescope have been spectacularly successful at identifying infrared excess emission associated with planetary debris disks around A- through K-type stars. The excesses at $70 \mu \mathrm{m}$ are associated with cool dust located at distances from the stars analogous to the position of the Kuiper Belt (KB) in the solar system (Moro-Martín et al. 2008), although much larger numbers of objects must lie in these exo-KBs to account for the detected level of emission (Trilling et al. 2008; Hillenbrand et al. 2008; Carpenter et al. 2009). The most sensitive studies find $70 \mu \mathrm{m}$ excesses around $\sim 15 \%-20 \%$ of mature solar-type stars over the entire $10 \mathrm{Myr}$ to $10 \mathrm{Gyr}$ age range (Trilling et al. 2008). The excesses at $24 \mu \mathrm{m}$ are generally associated with warmer dust and disappear relatively quickly as the host star ages; about $30 \%$ of the stars of the Pleiades age ( $\sim 120 \mathrm{Myr})$ show excess emission (Sierchio et al. 2010$)$ whereas by the age of Praesepe ( $\sim 600 \mathrm{Myr})$, the $24 \mu \mathrm{m}$ excesses have almost completely disappeared (Meyer et al. 2008; Gáspár et al. 2009). Because the expected lifetimes of the debris dust grains are much shorter than the ages of the stars, it is inferred that the dust originates from collisional activity in reservoirs of planetesimals left over from the planet formation process (hence the term debris dust). To sustain the dust production, it is necessary that large planetesimals (1000 km sized) or unseen planets stir the planetesimals so they continue to collide with each other. Planets are also responsible for constraining the planetesimals in some zones and clearing them from others, thus determining much of the structure of the debris system. The way the patterns of debris disks' activity decay with age is consistent with the expectation that the inner zones of a planetary system have relatively short dynamical timescales, whereas dynamical activity unfolds slowly at the distance of the KB.

A highlight of the recent surveys is the first detection of debris disks around stars with planets (Beichman et al. 2005;
Moro-Martín et al. 2007a). These planets orbit within several AU of their parent star, whereas the cold dust emitting the observed far-IR radiation generally resides tens of AU away. Despite the separation between the dust and planets, it is still possible for the planet to shape the structure of the dust (and planetesimals) disk. In the multiple-planet system HD 38529, for example, secular resonances excited by planets at 0.13 and 3.7 AU create regions at tens of AUs that are unstable for orbiting planetesimals (Moro-Martín et al. 2007b).

Several additional systems have been identified as having both multiple planets (capable of exciting secular resonances) and orbiting debris (indicating the presence of planetesimals; Bryden et al. 2009; Su et al. 2009). Presumably, there are many more stars with debris-disk excesses that also harbor multiple but undiscovered planets. Detailed studies of the known examples can reveal aspects of their behavior that help us understand the diversity of planetary systems. In this paper, we study four of these systems: HD 128311, HD 202206, HD 82943, and HR 8799. In Section 2, we describe the planet and debris dust detections for each one of these systems, and present new Spitzer Infrared Spectrograph detections for the debris disks around HD 202206 and HD 82943. In Section 3, we use a radiative transfer model to identify the range of parameters (dust mass and dust location) that would fit the observed spectral energy distribution (SED). Due to the high fractional luminosity, the grain-grain collisional timescale is shorter than the Poynting-Robertson (P-R) timescale for all these systems and therefore we expect the dust to trace the location of the dust-producing planetesimals. In Section 4, we use a dynamical model to assess the long-term orbital stability of the putative dust-producing planetesimals, taking into account the effect of secular resonances. Putting together the results from the SED and dynamical analysis, in Section 5 we discuss the potential location of the dust-producing planetesimal belts. 

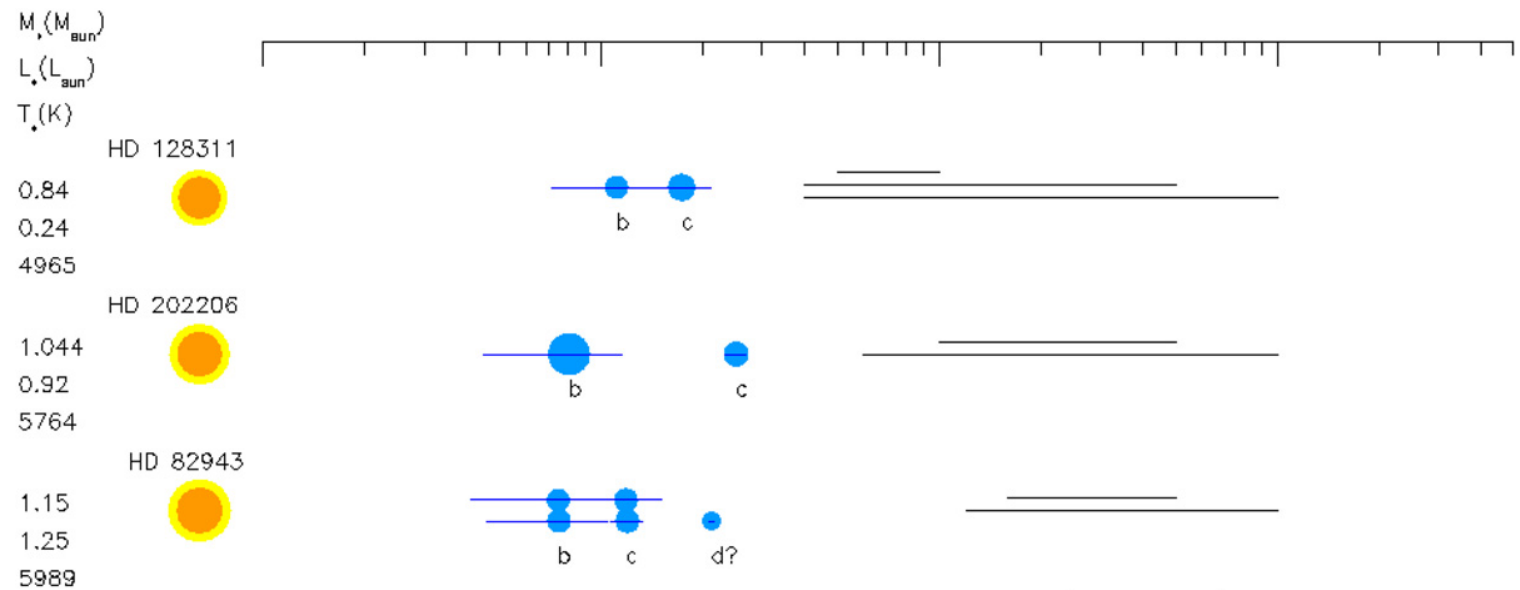

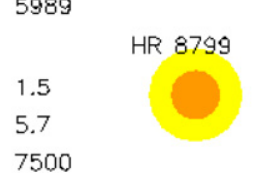

7500

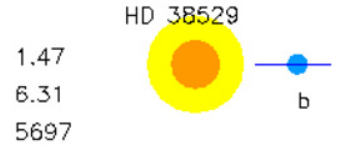

5697

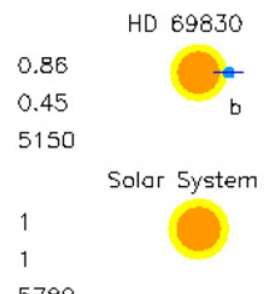

5780
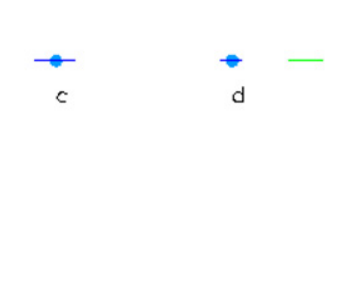

cold component

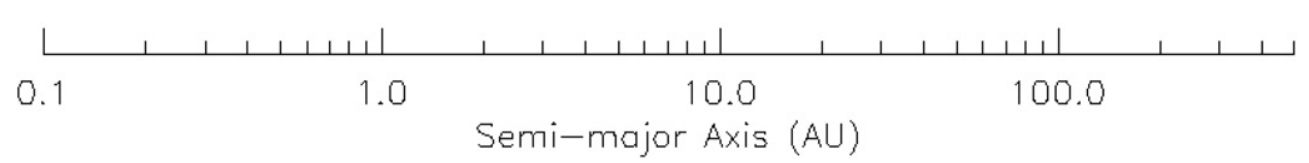

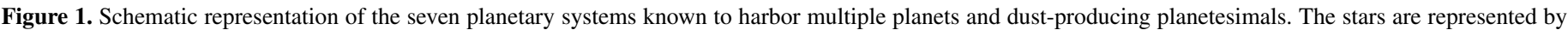

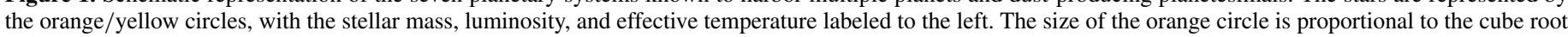

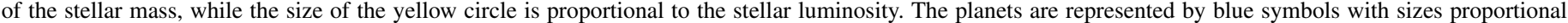

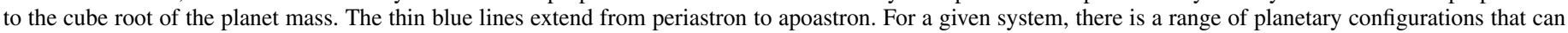

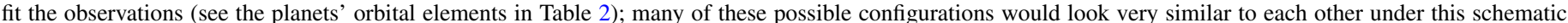

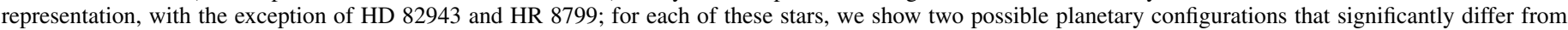

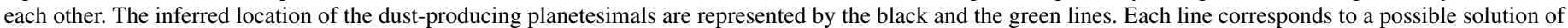

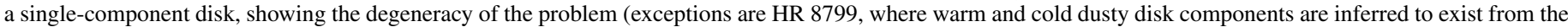

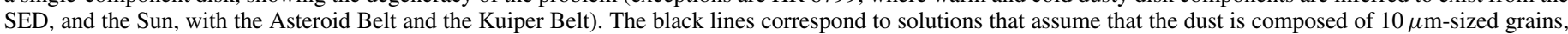

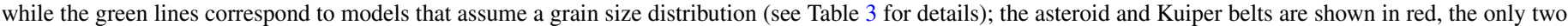
planetesimal belts that have been directly detected.

\section{DEBRIS DISKS DETECTED IN MULTIPLE-PLANET SYSTEMS}

Including the solar system, 19 planetary systems are currently known to have both orbiting debris and planets (Bryden et al. 2009), and while the majority are single-planet systems, seven of them are known to harbor multiple planets. These systems are described in Figure 1 and Table 3. Figure 1 also shows the locations of the planetesimal belts derived either from work in the literature or in this paper. Our estimates arise from SED fitting together with dynamical models that study the effects imposed by the planets on the stability of the planetesimals. In this section, we describe the infrared excesses, the planet detections, and the dynamical models for the planet orbits.

For these multi-planet systems, the planet-dust interaction has previously been studied in the case of the solar system,
HD 38529 (Moro-Martín et al. 2007b), and HD 69830 (Lovis et al. 2006; Lisse et al. 2007). In this paper, we study the remaining four systems: HD 128311, HD 202206, HD 82943, and HR 8799.

\subsection{Observations of HD 128311}

HD 128311 is a K0 star located at $16.57 \mathrm{pc}$, with $T_{\text {eff }}=$ $4965 \mathrm{~K}, M_{*}=0.84 M_{\odot}$, and a metallicity of $[\mathrm{Fe} / \mathrm{H}]=-0.04$ (Saffe et al. 2008). King et al. (2003) identified this star as a possible member of the UMa moving group, suggesting an age of $\sim 500 \mathrm{Myr}$, consistent with the estimate of 390-410 Myr by Saffe et al. (2005). The chromospheric activity index reported by Gray et al. (2003) indicates an age of $\sim 560 \mathrm{Myr}$, using the calibration of Mamajek \& Hillenbrand (2008). Barnes (2007) found an age of $350 \mathrm{Myr}$ from gyrochronology. All of these 
Table 1

Spitzer Photometry

\begin{tabular}{lccccccc}
\hline \hline Star & \multicolumn{5}{c}{ Wavelength $(\mu \mathrm{m})$} \\
\cline { 2 - 7 } & $13.20^{\mathrm{a}}$ & $19^{\mathrm{a}}$ & 23.68 & $25^{\mathrm{a}}$ & $32.5^{\mathrm{a}}$ & $71.42^{\mathrm{b}}$ & 155.9 \\
\hline HD 128311 & $192.45 \pm 15.62$ & & $60.0 \pm 1.2$ & & $32.95 \pm 4.82$ & $23.5 \pm 3.3^{\mathrm{c}}$ & $50.0 \pm 35.0$ \\
HD 202206 & $57.57 \pm 3.80$ & $28.94 \pm 1.95$ & N/A & $17.22 \pm 1.16$ & $14.23 \pm 0.83^{\mathrm{c}}$ & $32.0 \pm 3.1^{\mathrm{c}}$ & $\mathrm{N} / \mathrm{A}$ \\
HD 82943 & $201.10 \pm 10.87$ & $98.29 \pm 6.99$ & $66.0 \pm 1.3$ & & $46.98 \pm 3.27$ & $133 \pm 5^{\mathrm{c}}$ & N/A \\
\hline
\end{tabular}

Notes. Fluxes are given in mJy. The uncertainties are $1 \sigma$.

${ }^{a}$ Synthetic fluxes calculated from the IRS spectrum using a square filter over the following wavelength ranges: $13.2 \mu \mathrm{m}(12.4-14 \mu \mathrm{m}), 19 \mu \mathrm{m}(18-20 \mu \mathrm{m})$, $25 \mu \mathrm{m}(24-26 \mu \mathrm{m})$, and $32.5 \mu \mathrm{m}(30-35 \mu \mathrm{m})$. The uncertainties in this case are calculated from the standard deviation of the flux within the wavelength bin.

b The $70 \mu \mathrm{m}$ flux is color corrected assuming a dust temperature of $50 \mathrm{~K}$ and a correction factor of 0.893 (from the Spitzer Users Manual).

c Measured flux is significantly above that expected from the stellar photosphere.

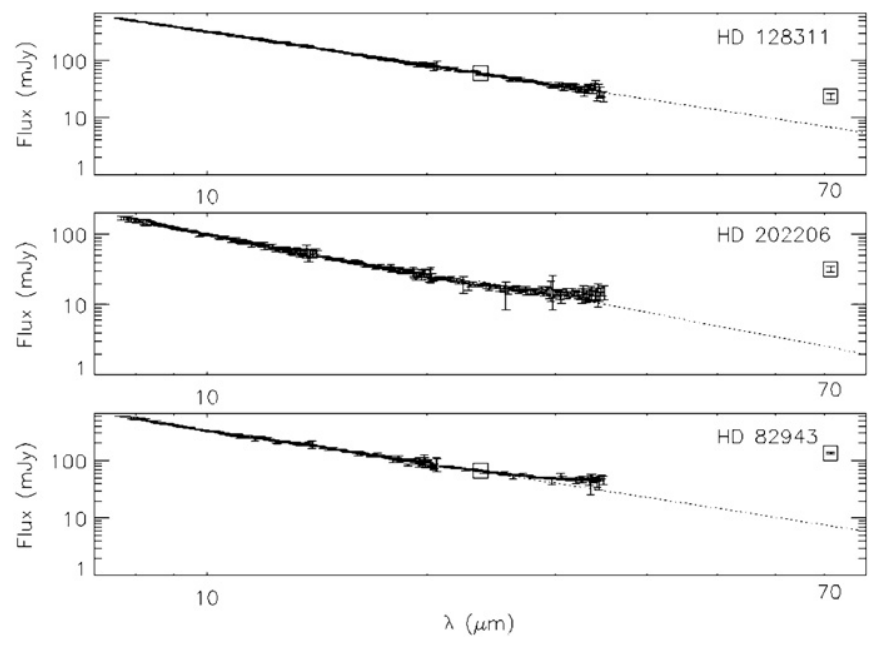

Figure 2. Spitzer observations of HD 128311, HD 202206, and HD 82943. The IRS spectrum is shown as a continuous line, while squares are the MIPS photometric points; in both cases, the error bars correspond to $1 \sigma$ uncertainties. The dotted line is the stellar photosphere (approximated as a blackbody).

determinations are consistent within their errors with an age of $500 \mathrm{Myr}$, which also lies at the lower end of the range from isochrone fitting (Valenti \& Fischer 2005).

\subsubsection{Debris Dust Detections}

HD 128311 was observed by a Spitzer Guaranteed Time Observation program specifically targeting planet-bearing stars. The Spitzer observations are shown in Figure 2 and Table 1. The star has a strong excess at $70 \mu \mathrm{m}$ (Beichman et al. 2005; Trilling et al. 2008), and no excess was detected with MIPS at $24 \mu \mathrm{m}$ (Trilling et al. 2008). No excess was seen in the IRS spectrum at 5-35 $\mu \mathrm{m}$ (Beichman et al. 2006). (HD 128311 has an MIPS $24 \mu \mathrm{m}$ flux of $60 \pm 1.2 \mathrm{mJy}$ ( $1 \sigma$ uncertainty), somewhat lower than but consistent with the flux expected from a $K$-band extrapolation (64 mJy)).

Excesses at $70 \mu \mathrm{m}$ are relatively common. Hillenbrand et al. (2008) and Carpenter et al. (2009) report them for 6\%-10\% of the 328 FGK stars in the FEPS sample, while the generally deeper (relative to the photospheric level) measurements of Trilling et al. (2008) find them in $16.4 \% \pm 2.9 \%$ of the FGK stars in their sample. However, HD 128311 may be exceptional in not having an excess at $33 \mu \mathrm{m}$, because only 3 out of 152 FGKM stars surveyed by Lawler et al. (2009) showed a $70 \mu \mathrm{m}$ excess with no corresponding $33 \mu \mathrm{m}$.

\subsubsection{Planet Detections}

Radial-velocity monitoring of HD 128311 has led to the discovery of two planets. We found that the orbital solution in Vogt et al. (2005) is not stable. In this paper, we have considered the following two orbital solutions (see planetary parameters in Table 2):

1. Fit Al is the solution in Butler et al. (2006), with the two planets near the 2:1 mean motion resonance (MMR).

2. Fit A2 (labeled as fit I in Goździewski \& Konacki 2006) corresponds to the best-fit, co-planar solution lying at the border of an island of stability related to the corotation of apsides, with the planets also in the 2:1 MMR.

Goździewski \& Konacki (2006) suggested that the radialvelocity data can also be explained by two planets in a non-coplanar 1:1 MMR. In the present paper, we do not consider this somewhat exotic solution, as we assume that the protoplanetary flattened disk results in a system where the planets and the dust-producing planetesimals are on the same plane.

Direct planet searches in the outer planetary system with the MMT (Biller et al. 2007) and VLT/NACO (Eggenberger et al. 2007) have led to non-detections, however, they can set no constraints on the presence of planets smaller than $10 M_{\text {Jup }}$.

\subsection{Observations of HD 202206}

HD 202206 is a metal-rich G6 V star located at 46.3 pc, with $T_{\text {eff }}=5765 \mathrm{~K}, M_{*}=1.15 M_{\odot}$, a metallicity of $[\mathrm{Fe} / \mathrm{H}]$ $=0.37 \pm 0.07$, and a stellar age of $5.6 \pm 1.2 \mathrm{Gyr}$ (Udry et al. 2001); Saffe et al. 2005 estimate an age of 4.2 Gyr.

\subsubsection{Debris Dust Detections}

HD 202206 was observed by a Spitzer Cycle 4 General Observer program targeting planet-bearing stars that were missed by the earlier Guaranteed Time Observations. The Spitzer observations are shown in Figure 2; Table 1 summarizes the (synthetic-)photometry. A strong excess was detected at $70 \mu \mathrm{m}$, a factor of 13 above the stellar photosphere (Bryden et al. 2009). In this paper, we present new IRS observations which show emission in excess of the stellar photosphere for $\lambda>25 \mu \mathrm{m}$ (see also S. E. Dodson-Robinson 2010, in preparation). No $24 \mu \mathrm{m}$ observations were made. We use the new IRS spectrum to calculate fluxes in four synthetic bands.

\subsubsection{Planet Detections}

From radial-velocity monitoring, Correia et al. (2005) concluded there are two planets in this system: a very massive 
Table 2

Planetary and Stellar Parameters for the Dynamical Simulations

\begin{tabular}{|c|c|c|c|c|c|c|c|c|c|c|}
\hline Planet & $\begin{array}{c}M_{*} \\
\left(M_{\odot}\right) \\
\end{array}$ & $\begin{array}{c}\left(I, \Omega^{\prime}\right) \\
(\mathrm{deg}, \mathrm{deg}) \\
\end{array}$ & $\begin{array}{l}M_{\text {planet }} \\
\left(M_{\text {Jup }}\right) \\
\end{array}$ & $\begin{array}{c}a \\
(\mathrm{AU}) \\
\end{array}$ & $e$ & $\begin{array}{c}i \\
(\mathrm{rad}) \\
\end{array}$ & $\begin{array}{c}\Omega \\
(\mathrm{rad}) \\
\end{array}$ & $\begin{array}{c}\omega \\
(\mathrm{rad}) \\
\end{array}$ & $\begin{array}{c}M \\
(\mathrm{rad})\end{array}$ & Ref \\
\hline $\begin{array}{l}\text { HD } 128311 \\
\text { Fit A1 }\end{array}$ & 0.84 & $(90, \ldots)$ & & & & & & & & B06 \\
\hline $\mathrm{b}$ & & & 2.19 & 1.10 & 0.25 & 0 & 0 & 1.93732 & 2.89 & \\
\hline $\mathrm{c}$ & & & 3.22 & 1.76 & 0.17 & 0 & 0 & 3.49066 & 0.068 & \\
\hline $\begin{array}{l}\text { HD } 128311 \\
\text { Fit A2 }\end{array}$ & 0.84 & $(90, \ldots)$ & & & & & & & & G06 \\
\hline $\mathrm{b}$ & & & 1.606 & 1.112 & 0.359 & 0 & 0 & 1.24931 & 4.74241 & \\
\hline $\mathrm{c}$ & & & 3.178 & 1.732 & 0.214 & 0 & 0 & 0.221831 & 3.32014 & \\
\hline $\begin{array}{l}\text { HD } 202206 \\
\text { Fit B1 }\end{array}$ & 1.044 & $(90, \ldots)$ & & & & & & & & $\mathrm{C} 09$ \\
\hline $\mathrm{b}$ & & & 16.59 & 0.8050 & 0.439 & 0 & 0 & 2.82412 & 1.35158 & \\
\hline $\mathrm{c}$ & & & 2.179 & 2.5113 & 0.071 & 0 & 0 & 1.36467 & 2.95153 & \\
\hline $\begin{array}{l}\text { HD } 82943 \\
\text { Fit C1 }\end{array}$ & 1.15 & $(90, \ldots)$ & & & & & & & & G06 \\
\hline $\mathrm{b}$ & & & 1.461 & 0.748 & 0.448 & 0 & 0 & 2.21343 & 6.26975 & \\
\hline $\mathrm{c}$ & & & 1.728 & 1.186 & 0.268 & 0 & 0 & 2.41466 & 5.87914 & \\
\hline $\begin{array}{l}\text { HD } 82943 \\
\text { Fit C2 }\end{array}$ & 1.15 & $(90, \ldots)$ & & & & & & & & G06 \\
\hline $\mathrm{b}$ & & & 1.679 & 0.751 & 0.386 & 0 & 0 & 2.06088 & 0.04625 & \\
\hline $\mathrm{c}$ & & & 1.867 & 1.197 & 0.110 & 0 & 0 & 2.52148 & 6.02557 & \\
\hline $\mathrm{d}$ & & & 0.487 & 2.125 & 0.018 & 0 & 0 & 2.00032 & 1.39207 & \\
\hline HD 82943 & 1.15 & & & & & & & & & B08 \\
\hline Fit C3 & & $(90, \ldots)$ & & & & & & & & \\
\hline $\mathrm{b}$ & & & 1.703 & 0.745 & 0.361 & 0 & 0 & 2.30942 & 6.11127 & \\
\hline $\mathrm{c}$ & & & 1.747 & 1.200 & 0.190 & 0 & 0 & 3.35121 & 5.20335 & \\
\hline d & & & 0.351 & 1.912 & 0.078 & 0 & 0 & 2.0279 & 0.482234 & \\
\hline HR 8799 & 1.5 & & & & & & & & & F10 \\
\hline Fit D1 & & $(0, \ldots)$ & & & & & & & & \\
\hline b & & & 7 & 67.91 & 0 & 0 & 0 & 0 & 5.80078 & \\
\hline $\mathrm{c}$ & & & 10 & 37.97 & 0 & 0 & 0 & 0 & 3.96172 & \\
\hline $\mathrm{d}$ & & & 10 & 23.42 & 0.09 & 0 & 0 & 6.00393 & 2.19911 & \\
\hline HR 8799 & 1.5 & & & & & & & & & R09 \\
\hline Fit D2 & & $(25,20)$ & & & & & & & & \\
\hline $\mathrm{b}$ & & & 5 & 71.2002 & 0 & 0 & 0 & 0 & 0.789567 & \\
\hline $\mathrm{c}$ & & & 7 & 41.1437 & 0 & 0 & 0 & 0 & -1.13722 & \\
\hline $\mathrm{d}$ & & & 7 & 24.4592 & 0 & 0 & 0 & 0 & -3.13463 & \\
\hline HR 8799 & 1.5 & & & & & & & & & R09 \\
\hline Fit D3 & & $(20,45)$ & & & & & & & & \\
\hline $\mathrm{b}$ & & & 5 & 68.3211 & 0 & 0 & 0 & 0 & 0.322337 & \\
\hline $\mathrm{c}$ & & & 7 & 40.4333 & 0 & 0 & 0 & 0 & -1.53703 & \\
\hline $\mathrm{d}$ & & & 7 & 24.7391 & 0 & 0 & 0 & 0 & -3.59566 & \\
\hline HR 8799 & 1.445 & & & & & & & & & G09 \\
\hline Fit D4 & & $(15.5,11.2)$ & & & & & & & & \\
\hline $\mathrm{b}$ & & & 8.022 & 68.448 & 0.008 & 0 & 0 & 5.38591 & 3.3458 & \\
\hline $\mathrm{c}$ & & & 11.87 & 39.646 & 0.012 & 0 & 0 & 6.17550 & 0.698655 & \\
\hline $\mathrm{d}$ & & & 8.891 & 24.181 & 0.075 & 0 & 0 & 2.51991 & 2.22704 & \\
\hline HR 8799 & 1.611 & & & & & & & & & G09 \\
\hline Fit D5 & & $(11.4,357.2)$ & & & & & & & & \\
\hline $\mathrm{b}$ & & & 9.708 & 67.661 & 0.014 & 0 & 0 & 0.517857 & 2.16072 & \\
\hline $\mathrm{c}$ & & & 7.963 & 31.045 & 0.248 & 0 & 0 & 4.24831 & 2.77158 & \\
\hline $\mathrm{d}$ & & & 7.397 & 30.777 & 0.267 & 0 & 0 & 6.08038 & 5.69850 & \\
\hline
\end{tabular}

Notes. Planetary parameters used in the dynamical simulations in Section 4. $a$ and $e$ are the semimajor axis and eccentricity of the planet; in all cases, the orbits considered are co-planar $(i=0)$; $\omega$ is the longitude of periastron; $\Omega$ is the longitude of the ascending node; $M$ is the mean anomaly. The orientation of the orbit is given by angles $I$ and $\Omega^{\prime}: I$ is the inclination of the orbital plane with respect to the plane of the sky, and $\Omega^{\prime}$ is the angle between the north direction and the line of nodes (measured toward the east—see Figure 1 in Reidemeister et al. 2009). References are: B06: Butler et al. 2006; G06: Goździewski \& Konacki 2006; C09: Couetdic et al. 2009; F10: Fabrycky \& Murray-Clay 2010; B08: Beauge et al. 2008; R09: Reidemeister et al. 2009; G09 Goździewski \& Migaszewski 2009. 
17.5 $M_{\text {Jup }}$ inner planet and a less massive $2.4 M_{\text {Jup }}$ outer planet. This system is particularly interesting because of the high mass of the innermost planet (or brown dwarf). If it formed in the circumstellar protoplanetary disk, its existence would imply that such disks can be extremely massive, whereas if it formed like a stellar companion this system would be the only known example of a circumbinary planet orbit. In the latter situation, Nelson (2003) predicted that the interaction of the outermost planet with the viscous circumbinary disk could have been responsible for its inward migration and resonance trapping.

From stability considerations, Correia et al. (2005) suggested that the system is in an island of stability around the 5:1 MMR, with $M \sin i$ of $17.428 M_{\text {Jup }}$ and $2.436 M_{\text {Jup }}$, semimajor axes of $0.830 \mathrm{AU}$ and 2.542 AU, and eccentricities of 0.435 and 0.267 , for HD $202206 \mathrm{~b}$ and c, respectively. This solution has a $\left(\chi_{v}{ }^{2}\right)^{1 / 2}$ of 1.67 and is stable over a 5 Gyr timescale. However, using $n$ body analysis that takes into account stability considerations and is well suited for multi-planet systems in low-order MMRs, Goździewski et al. (2006) concluded that the solution by Correia et al. (2005) is a local minimum. Assuming a co-planar system, their best fit (with rms $=9.98 \mathrm{~m} \mathrm{~s}^{-1}$ and $\left(\chi_{\nu}{ }^{2}\right)^{1 / 2}=1.53$ ) has $M \sin i$ of $17.624 M_{\mathrm{Jup}}$ and $2.421 M_{\mathrm{Jup}}$, semimajor axes of $0.831 \mathrm{AU}$ and $2.701 \mathrm{AU}$, and eccentricities of 0.433 and 0.255 , for HD 202206 b and c, respectively. (Goździewski et al. (2006) noted that an unexpected non-co-planar solution is also possible, where the eccentricity of the innermost largest planet varies with larger amplitude than that of the outermost planet, while the inclination of the latter can assume almost any value). In a more recent paper, Couetdic et al. (2009) used frequency map analysis and updated radial-velocity data to explore the long-term stability of a wide range of possible orbital solutions. Adopting an updated stellar mass of $1.044 M_{\odot}$, they found acceptable co-planar configurations for inclinations between $30^{\circ}$ and $90^{\circ}$ with respect to the line of sight and favored an edgeon co-planar solution in which the bodies are in the 5:1 MMR (with $\left(\chi_{v}{ }^{2}\right)^{1 / 2}=1.4136$ ). This solution has $M \sin i$ of $16.59 M_{\text {Jup }}$ and 2.179 $M_{\text {Jup }}$, semimajor axes of $0.8053 \mathrm{AU}$ and 2.49 AU, and eccentricities of 0.431 and 0.104 , for HD 202206 b and c, respectively. It is stable for more than $5 \mathrm{Gyr}$ and differs from the solutions above in that the eccentricity of planet $\mathrm{c}$ is lower, allowing more stable regions outside the resonances. Couetdic et al. (2009) explored the stability of test particles in the above configuration identifying two possible niches for stability: $a<0.12$ and $a>6.5 \mathrm{AU}$ (for an integration time of 16,000 years). Couetdic et al. (2009) noted that the solution above has a high resonant mode amplitude that will likely be dampened by dissipative forces.

1. Fit B1 in Table 2 is the edge-on co-planar configuration favored by Couetdic et al. (2009); this solution is close to the center of libration and has $\left(\chi_{v}^{2}\right)^{1 / 2}=1.553$.

The radial-velocity data acquired so far cannot exclude the presence of a Neptune-sized planet between $0.06 \mathrm{AU}$ and $0.12 \mathrm{AU}$, or a $10 M_{\oplus}$ planet between $0.02 \mathrm{AU}$ and $0.12 \mathrm{AU}$, or a $0.5 M_{\text {Jup }}$ mass planet outside 6.5 AU. No planetary companions have been found by direct imaging (a source detected by Chauvin et al. 2006 was a background object).

\subsection{Observations of HD 82943}

HD 82943 is a G0 V star located at $27.46 \mathrm{pc}$, with $T_{\text {eff }}=$ $5989 \mathrm{~K}, M_{*}=1.15 M_{\odot}$, and $[\mathrm{Fe} / \mathrm{H}]=0.26$ (Sousa et al. 2008). Consistent measurements of the chromospheric activity have been reported by Wright et al. (2004), Gray et al. (2006), and Saffe et al. (2005). Using the calibration of Mamajek \& Hillenbrand (2008), they imply an age of 5 Gyr.

\subsubsection{Debris Dust Detections}

HD 82943 was observed by a Spitzer Guaranteed Time Observation program targeting nearby late $\mathrm{F}, \mathrm{G}$, and early $\mathrm{K}$ stars. The Spitzer observations are shown in Figure 2; Table 1 summarizes the (synthetic-)photometry. Trilling et al. (2008) found a strong excess emission at $70 \mu \mathrm{m}$ and no excess at $24 \mu \mathrm{m}$, deriving a minimum characteristic dust temperature of $69 \mathrm{~K}$ (corresponding to $22 \mathrm{AU}$ if assuming blackbody grains), and a fractional luminosity of $L_{\text {dust }} / L_{\text {star }} \sim 10^{-4}$. In this paper, we present new IRS observations that show how the spectrum rises above the stellar photosphere for $\lambda>26 \mu \mathrm{m}$.

\subsubsection{Planet Detections}

From radial-velocity observations, Mayor et al. (2004) announced the presence of two planets around HD 82943. FerrazMello et al. (2005) found that the orbital solution in the discovery paper is unstable and that the radial-velocity observations can be fitted with a stable, co-planar solution in which the planets are locked in the 2:1 MMR. Goździewski \& Konacki (2006) revisited the orbital solution modeling data from CORALIE (Mayor et al. 2004) and from Keck-HIRES (Lee et al. 2006), confirming that the co-planar 2:1 MMR solution fits the observations. They also found a stable, non-co-planar solution in a 1:1 MMR, but as we mentioned above in this paper we only consider co-planar configurations. The dynamical maps in Goździewski \& Konacki (2006) revealed that near their unstable, best-fit solution there are two narrow islands of stability associated with the 2:1 MMR (see orbital elements in Table 2):

1. Fit C1 (labeled fit V in Goździewski \& Konacki 2006) lies in one of the two islands and corresponds to their bestfit, rigorously stable, two-planet solution, characterized by co-rotation of the apsidal lines. (We found that the second solution they identify in their Figure 9 becomes unstable after 27.8 Myr and therefore we do not consider it in this paper; this solution lies at the very edge of the second island of stability of the 2:1 MMR, where slight differences in the initial conditions can result in a very different dynamical evolution).

Goździewski \& Konacki (2006) and Beauge et al. (2008) discussed the inadequacy of the two-body solution and speculated on the presence of a third planet to account for the large rms and the lack of convergence of some of the orbital elements (eccentricity and longitude of periapsis) as the number of radial-velocity data points increases. In this paper, we have also considered the following stable, three-planet, co-planar solutions (see orbital elements in Table 2):

1. Fit $C 2$ is the solution in Goździewski \& Konacki (2006), in which planets $b$ and $c$ are in the 2:1 MMR while the outermost planet $\mathrm{d}$ is in a low eccentricity non-resonant orbit.

2. Fit $C 3$ is the solution in Beauge et al. (2008), in which the planets are in a Laplace $1 \mathrm{~d}: 2 \mathrm{c}: 4 \mathrm{~b}$ MMR in a double asymmetric apsidal corotation resonance.

\subsection{Observations of HR 8799}

HR 8799 is a metal-poor A5 V star located at 39.4 pc, with $T_{\text {eff }}=7500 \mathrm{~K}, M_{*}=1.5 M_{\odot}$, a metallicity of $[\mathrm{Fe} / \mathrm{H}]=-0.55$. Marois et al. (2008) estimate a stellar age of 30-160 Myr; 
Moya et al. (2010) argue that the age is still unconstrained, with astroseismology analysis favoring an older age of $\sim 1 \mathrm{Gyr}$, but requiring a better determination of the rotation velocity of the star.

\subsubsection{Debris Dust Detections}

HR 8799 was observed by Spitzer under programs 50175 and 530; images and an SED analysis were presented in Su et al. (2009). Deep IRS and MIPS observations (including the MIPS-SED mode from 55 to $95 \mu \mathrm{m}$ - a wavelength range critical to constrain the shape of the SED), revealed a spatially resolved disk at 24 and $70 \mu \mathrm{m}$, whose outer boundary can be traced beyond $1000 \mathrm{AU}$. The excess dust emission can be detected in the IRS spectra starting below $20 \mu \mathrm{m}$ (less deep IRS observations are discussed in Chen et al. 2009) and also at $160 \mu \mathrm{m}$. Unresolved debris-disk detections exist at 60 and $90 \mu \mathrm{m}$ (with Infrared Space Observatory (ISO); Moór et al. 2006), at $850 \mu \mathrm{m}$ (with James Clerk Maxwell telescope (JCMT)/SCUBA; Williams \& Andrews 2006), and at $1.2 \mathrm{~mm}$ (Sylvester et al. 1996). Preliminary analysis shows that the debris disk is spatially resolved with APEX/LABOCA at $870 \mu \mathrm{m}$ (Kalas et al. 2010).

\subsubsection{Planet Detections}

Three distant planets have been detected by direct imaging at projected separations of $68 \mathrm{AU}, 38 \mathrm{AU}$, and $24 \mathrm{AU}$ (for HR 8799 b, c, and d, respectively-Marois et al. 2008; Lafrenière et al. 2009); the planets' long orbital periods and the short baseline of the observations make the determination of the orbital parameters still uncertain. It is possible to estimate the masses of the directly detected planets from their luminosities by using evolutionary cooling models (assuming the age of the system is known). However, current models are discrepant and, in the case of HR 8799, the age of the system is uncertain, making the planets' masses poorly constrained; lower limits to the masses of HR $8799 \mathrm{~b}$, c, and d are in the ranges of 5-11, 7-13, and 7-13 $M_{\text {Jup }}$, with nominal masses of 7, 10, and $10 M_{\text {Jup }}$, respectively (Marois et al. 2008; Reidemeister et al. 2009).

Fabrycky \& Murray-Clay (2010) found that many orbital solutions are unstable, including the face-on circular configuration (for any range of reasonable planet masses). They tried to determine the system parameters by simultaneously fitting the astrometry (positions and proper motions), the planets' luminosities (that constrain the range of possible planets' masses), and the requirement that the system is stable for at least 30-160 Myr (the age of the system as estimated by Marois et al. 2008).

1. Fit Dl is the face-on, co-planar solution identified by Fabrycky \& Murray-Clay (2010) that is stable for > $160 \mathrm{Myr}$ even if the planets' masses are up to 1.9 times their nominal values; in this solution, the planets are locked into a Laplace 1d:2c:4b MMR, allowing them to avoid close encounters (see orbital elements in Table 2).

Reidemeister et al. (2009) have also explored the stability of the HR 8799 system in the case of co-planar and initially circular orbits, where the orbital plane is allowed to have a range of inclinations $\left(I=0^{\circ}-40^{\circ}\right)$ and orientations $\left(\Omega^{\prime}=0^{\circ}-180^{\circ}\right)$. Here, $I$ is the angle between the angular momentum vector and the vector toward the observer, and $\Omega^{\prime}$ is the angle between the north direction and the line of nodes (measured toward the east—see Figure 1 in Reidemeister et al. 2009). They integrated a grid of models over $100 \mathrm{Myr}$ and identified as stable the configurations where the planets did not suffer close encounters during that period; these configurations (all co-planar) have $I>20^{\circ}$ and $\Omega^{\prime}=0^{\circ}-50^{\circ}$. We take into account the following considerations:

1. Figure 8 in Reidemeister et al. (2009), showing the models that fulfill the stability criteria (i.e., no close encounters during $100 \mathrm{Myr}$ ).

2. Figure 9 in Reidemeister et al. (2009), showing the models that fit the observed astrometry.

3. Inclination constraints of the equatorial plane of the star derived from the comparison of the measured rotational velocity $v \sin i$ and the expected rotational velocity for an A5 star: $I=7^{\circ}-22^{\circ}$ (Lafrenière et al. 2009).

4. Inclination constraints from astrometry: (1) assuming circular, co-planar orbits and fixing the stellar mass to $1.5 M_{\odot}$, but letting the orientation of the orbit float: $I \sim 20^{\circ}$ (Fabrycky \& Murray-Clay 2010) and (2) from observations with a 10 year baseline (using NICMOS archival data where the planets are detected): $I=13^{\circ}-23^{\circ}$ (Lafrenière et al. 2009).

5. Inclination constraints from the debris-disk morphology: (1) from Spitzer spatially resolved observations: $I<25^{\circ}$ (Su et al. 2009) and (2) from preliminary analysis of $870 \mu \mathrm{m}$ observations: $I>20^{\circ}$ (Kalas et al. 2010).

Based on the above considerations, we have adopted the following two additional non-face-on, co-planar configurations (see orbital elements in Table 2):

1. Fit $D 2$ with $\left(I=25^{\circ}, \Omega^{\prime}=20^{\circ}\right)$.

2. Fit D3 with $\left(I=20^{\circ}, \Omega^{\prime}=45^{\circ}\right)$.

As for the planets' masses, we initially considered: (1) the nominal masses of 7, 10, and $10 M_{\text {Jup }}$ for HR $8799 \mathrm{~b}$, c, and d, respectively and (2) the low mass case with 5, 7, and $7 M_{\text {Jup }}$ for HR 8799 b, c, and d, respectively. However, we found that when adopting nominal planet masses, the configurations D2 and D3 were unstable.

Based on the location of HR $8799 \mathrm{~b}$ in a pre-discovery 2007 image (Metchev et al. 2009), Fabrycky \& Murray-Clay (2010) ruled out stable co-planar configurations in which the innermost planet is very eccentric $(e>0.95)$ or retrograde. They also found very non-co-planar (non-resonant) configurations that are stable but we will not consider those because: (1) they imply we are observing the system at a special time and (2) it is more reasonable to assume that the planets and the planetesimals that produce the dust are co-planar because they formed out of a flattened disk.

In a recent study of HR 8799, Goździewski \& Migaszewski (2009) used a self-consistent, $n$-body analysis that takes into account stability considerations to carry out a quasi-global search for stable, co-planar planetary configurations that can fit the astrometric data and the astrophysical mass constraints for the star and the planets. In their study, the planets and stellar masses are free parameters and are allowed to vary within their $1 \sigma$ range, with $M_{b}=7_{-2}^{+4} M_{\text {Jup }}, M_{c}=10 \pm 3 M_{\text {Jup }}, M_{d}=$ $10 \pm 3 M_{\mathrm{Jup}}$, and $M_{*}=1.5 \pm 0.3 M_{\odot}$. As in Fabrycky \& MurrayClay (2010) and Reidemeister et al. (2009), the goal of their study is to use the requirement of stability to identify longlived configurations that may lie close to unstable best-fitting solutions, mitigating the errors introduced by the short baseline of the observations. Goździewski \& Migaszewski (2009) found two long-term, stable, non-face-on, co-planar solutions (see orbital elements in Table 2): 
1. Fit $D 4\left(I=15.5, \Omega^{\prime}=11.2\right)$ : inside a small island of regular motion; the planets are in the $1 \mathrm{~d}: 2 \mathrm{c}: 4 \mathrm{~b}$ MMR; the solution becomes unstable after $400 \mathrm{Myr}$.

2. Fit $D 5\left(I=11.4, \Omega^{\prime}=357.2\right)$ : also inside a small island of stable motion; the inner two planets are in the 1c:1d MMR, and the outermost planet is in a narrow island close to the (1c:1d):3b MMR; the inner planets are stable for $>3 \mathrm{Gyr}$, while the three-planet system has a regular motion only during the first $600 \mathrm{Myr}$.

Table 2 lists the orbital elements of the five planetary configurations (fits D1-D5) we have adopted for our test particle simulations of HR 8799 in Section 4.4. Our numerical runs last $160 \mathrm{Myr}$ (the upper limit for the age of HR 8799 estimated by Marois et al. 2008); there is no guarantee that the systems will remain stable on longer timescales.

\section{SED MODELING}

We modeled the excess dust emission using the radiative transfer code developed by Wolf \& Hillenbrand (2003). For a study on how the SEDs depend on the grain composition, we refer to Wolf \& Hillenbrand (2003) and Moro-Martín et al. (2005). For the grain properties, we assumed a silicate composition with optical constants from Weingartner \& Draine (2001), and two assumptions for the particle radius $(b)$ :

1. A single grain size of $b=10 \mu \mathrm{m}$. This size was chosen because these grains emit efficiently at $70 \mu \mathrm{m}$. We favor this scenario because it represents large grains in general, where the grains would be located at the blackbody equilibrium distance from the central star (modulo bulk optical properties). Given the lack of spectral features in most debris disks (see, e.g., Beichman et al. 2006; Lawler et al. 2009), it is inferred that small grains with super-thermal behavior are mostly absent, therefore, this single grain size scenario provides a plausible limiting case for the placement of the grains.

2. A particle size distribution following a power law. The placements of the emitting zones are rather uncertain. In addition to the single grain size scenario above, the other bounding case would be given by assuming a power law down to the blow-out size. We used $n(b) \propto b^{-3.5}$, with $b_{\max }=10 \mu \mathrm{m}$ and $b_{\min }=b_{\text {blow }}$. The blow-out size, $b_{\text {blow }}$, is the grain radius for which $\beta$, the ratio between the radiation pressure force and the gravitational force, equals $1 / 2$; for spherical grains, $\beta=\frac{3 L_{*}}{16 \pi G M_{*} c} \frac{<Q_{\mathrm{pr}}>}{\rho b}=$ $5.7 \times 10^{-5} \frac{<Q_{\mathrm{pr}}>}{\rho b} \frac{L_{*}}{L_{\odot}} \frac{M_{\odot}}{M_{*}}$ (Burns et al. 1979), where $\rho$ and $b$ are the density and radius of the grain in cgs units and $Q_{\mathrm{pr}}$ is the radiation pressure coefficient, a measure of the fractional amount of energy scattered and/or absorbed by the grain. $Q_{\mathrm{pr}}$ is a function of the physical properties of the grain and the wavelength of the incoming radiation; the value we use, $\left\langle Q_{\mathrm{pr}}>\right.$, is an average integrated over the stellar spectrum.

The presence of larger and colder grains is not constrained by the Spitzer observations, so generally these models lead to lower limits of the dust disk mass.

We now discuss our assumptions for the disk geometry. We adopted a constant surface density disk $\left(\Sigma \propto r^{0}\right)$. The dust disk outer radius, $R_{\text {out }}$, cannot be determined from the Spitzer data alone. Given that the debris disks around HD 128311, HD 202206, and HD 82943 are not spatially resolved at $70 \mu \mathrm{m}$ (with a point-spread function (PSF) FWHM of $18^{\prime \prime}$ ), and that the debris disk around $\epsilon$ Eri (of similar spectral type) was well constrained by the Spitzer data to be $\sim 100$ AU (Backman et al. 2009), we considered three possible outer disk radii: $R_{\text {out }}=10 \mathrm{AU}, 50 \mathrm{AU}$, and $100 \mathrm{AU}$. To explore different belt widths, the inner disk radius, $R_{\mathrm{in}}$, is allowed to vary from the sublimation radius (where $T_{\text {sub }}=1550 \mathrm{~K}$ ) to $R_{\text {out }}$.

We further assumed that the dust disk is optically thin (which is supported by the low fractional luminosities observed for HD 128311, HD 202206, and HD 82943, with $L_{\text {dust }} / L_{\text {star }}<10^{-4}$; see Table 3). Under this scenario, only scattering, absorption, and reemission of stellar radiation by dust grains were taken into account (neglecting scattering and dust heating from the dust infrared radiation). With the parameters described above, we calculated the dust disk emission at 200 wavelengths from $3 \mu \mathrm{m}$ to $600 \mu \mathrm{m}$.

\subsection{HD 128311}

Beichman et al. (2006) modeled the IRS and MIPS $70 \mu \mathrm{m}$ data with a single population of $10 \mu \mathrm{m}$ amorphous silicate grains located at $R_{\text {dust }}>15 \mathrm{AU}\left(T_{\text {dust }}<50 \mathrm{~K}\right)$, while the modeling of the MIPS $24 \mu \mathrm{m}$ and $70 \mu \mathrm{m}$ data in Trilling et al. (2008) implied $R_{\text {dust }}>5.1 \mathrm{AU}\left(T_{\text {dust }}<106 \mathrm{~K}\right)$, with a fractional luminosity $L_{\text {dust }} / L_{\text {star }}=1.3-2.7 \times 10^{-5}$.

To model the dust emission, the stellar contribution needs to be subtracted from the observed SED. Because the slope of the IRS spectra of HD 128311 indicates there is no dust excess at $\lambda<34 \mu \mathrm{m}$, we pin the photosphere to the MIPS $24 \mu \mathrm{m}$ flux; for simplicity, the stellar emission was modeled as a blackbody with $T_{\text {eff }}=4965 \mathrm{~K}, L_{*}=0.24 L_{\odot}$, and a distance of $16.57 \mathrm{pc}$.

For a stellar mass of $M_{*}=0.84 M_{\odot}$, a grain density $\rho=$ $2.5 \mathrm{~g} \mathrm{~cm}^{-3}$, and adopting the optical constants of astronomical silicates, we find that the maximum $\beta$-value is 0.26 , i.e., there is no blow-out size because $\beta<0.5$.

Figure 3 shows the SED models compared to the SED observed by Spitzer. We calculated the synthetic photometry of each SED model using the MIPS and IRS filter profiles from the Spitzer Users Manual, with effective wavelengths at $13.2 \mu \mathrm{m}$, $23.68 \mu \mathrm{m}, 32.5 \mu \mathrm{m}, 71.42 \mu \mathrm{m}$, and $155.9 \mu \mathrm{m}$. Then, for each model we calculated $\chi^{2}$ and the $\chi^{2}$ probability, where the fit is done to the five photometric points in Table 1 with two free parameters $\left(R_{\min }\right.$ and $\left.M_{\text {dust }}\right)$. (Because the uncertainty in the $160 \mu \mathrm{m}$ flux is large, even though we are including it in the SED fit, in practice its contribution is negligible). The colors indicate the goodness of the fit. Figure 4 shows the parameter space of the SED models in Figure 3. The yellow area corresponds to combinations of $R_{\text {in }}$ and $M_{\text {dust }}$ that lead to a $\chi^{2}$ probability of $P\left(\chi^{2} \mid v\right)<0.683$, light blue is for $0.683<P\left(\chi^{2} \mid v\right)<0.9973$, and dark blue if for $P\left(\chi^{2} \mid v\right)>0.9973$. The modeling of the SED is degenerate. For the single grain size models, valid SED fits can be obtained for $R_{\text {out }}=10 \mathrm{AU}$ and $R_{\text {in }} \geqslant 5 \mathrm{AU} ; R_{\text {out }}=50 \mathrm{AU}$ and $R_{\text {in }} \geqslant 2 \mathrm{AU}$; and $R_{\text {out }}=100 \mathrm{AU}$ and $R_{\text {in }} \geqslant 2$ AU. In all three cases, narrow belts with widths of $10 \%$ the disk radius could also fit the observed SEDs. Here, we would like to emphasize the lower limits to $R_{\text {in }}$ because of two reasons: (1) for HD 128311, HD 202206, and HD 82943, the dust is located outside the orbits of the planets and, therefore, it is at the disk inner edge where the gravitational effects of the planets are stronger (i.e., it is at that location that there is a closer correspondence between the SED models and the dynamical models). (2) The narrowest belts might only be justified in the presence of additional planetary perturbers for which we have no evidence so far.

As we mentioned before, we favor the single grain size models above because they represent large grains in general, where the 
Table 3

Stars with Evidence of Harboring a Multi-planet system and Planetesimals

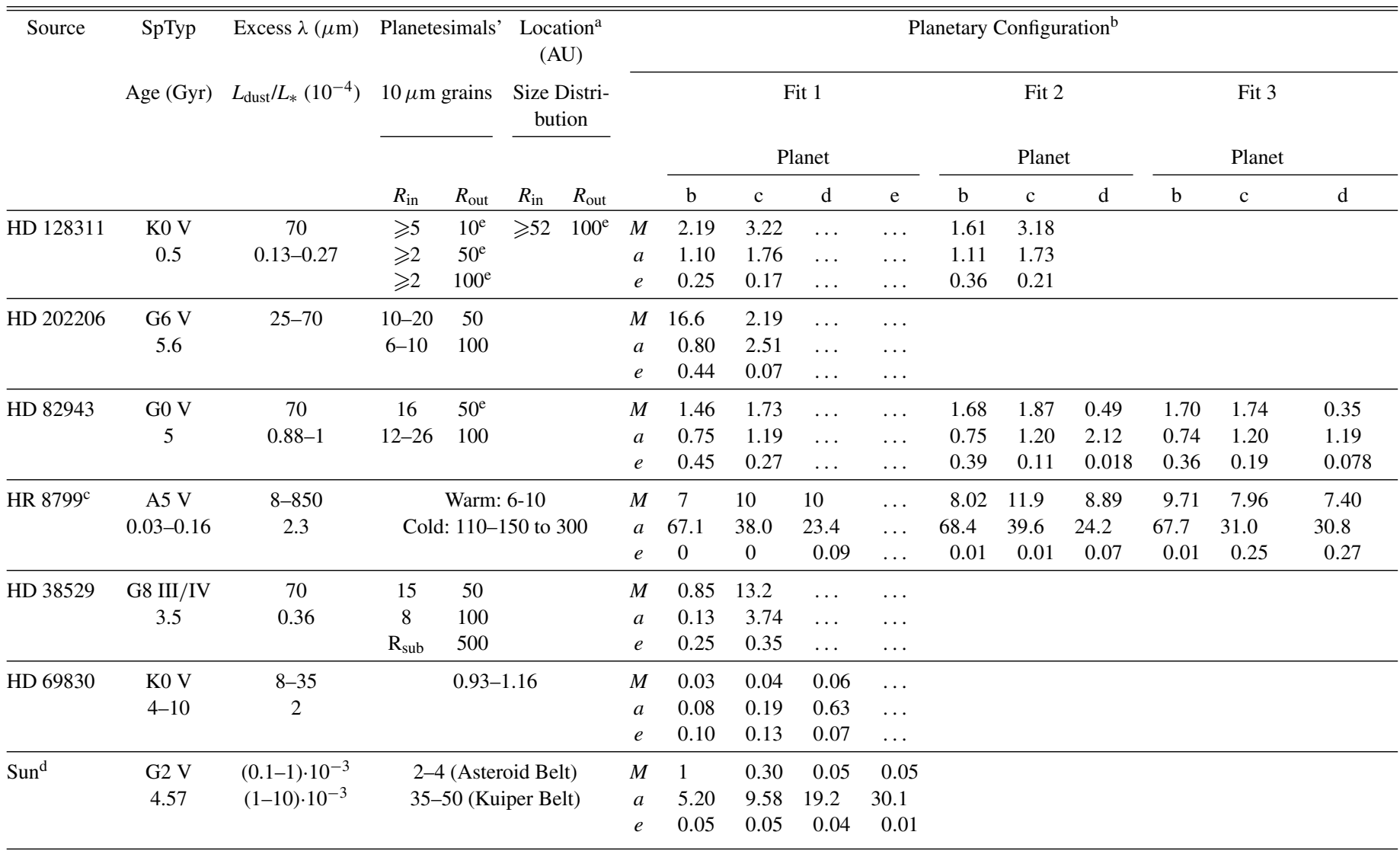

Notes.

a Inferred planetesimals' location. HD 128311: this work; HD 202206: this work; HD 82943: this work; HR 8799 : Su et al. (2009) and this work; HD 38529: Moro-Martín et al. 2007b; HD 69830: Lisse et al. 2007.

${ }^{\mathrm{b}}$ Planetary parameters: $M, a$, and $e$ are planet mass in $M_{\mathrm{Jup}}$, semimajor axis in AU and eccentricity, respectively. The rest of the orbital elements are listed in Table 2 . ${ }^{c}$ For HR 8799, this table only includes the planetary configurations where the planets have their nominal masses; the SED reveals the presence of a warm component and a cold component of the dust disk (from Su et al. 2009).

${ }^{\mathrm{d}}$ Fractional luminosities of asteroidal dust and Kuiper Belt dust from Dermott et al. (2002) and Stern (1996).

${ }^{\mathrm{e}}$ Narrow belts of width $10 \%$ of the disk radius give valid fits to the SED (but see discussion in Section 3.1).

grains would be located at the blackbody equilibrium distance from the central star. However, for completeness and to explore the other limiting case we have also considered the case of a grain size distribution. For these models, valid fits can be obtained for $R_{\text {out }}=100 \mathrm{AU}$ and $R_{\text {in }} \geqslant 52 \mathrm{AU}$. A small disk is ruled out for this grain size distribution. We can exclude the presence of a significant population of small grains unless the disk is large, $100 \mathrm{AU}$, in which case there would be a depletion of small grains inside $52 \mathrm{AU}$.

\subsection{HD 202206}

The slope of the IRS spectrum of HD 202206 indicates there is no dust excess at $\lambda<25 \mu \mathrm{m}$; therefore, we used the IRS synthetic photometry at $15-19 \mu \mathrm{m}$ to determine the photospheric emission level. The stellar emission was modeled as a blackbody with $T_{\text {eff }}=5764 \mathrm{~K}, L_{*}=0.92 L_{\odot}$, and a distance of $46.3 \mathrm{pc}$.

For a stellar mass of $M_{*}=1.044 M_{\odot}$, a grain density $\rho=$ $2.5 \mathrm{~g} \mathrm{~cm}^{-3}$ and adopting the optical constants of astronomical silicates, the blow-out size is $b_{\text {blow }}=0.5 \mu \mathrm{m}$.

For the SED modeling, we followed the same scheme as described above for HD 128311. Figure 5 (top) shows some of the SED models computed; overplotted are the Spitzer observa- tions. For each model, we calculated the synthetic photometry using the MIPS and adopted IRS filter profiles at effective wavelengths $13.2 \mu \mathrm{m}, 19 \mu \mathrm{m}, 25 \mu \mathrm{m}, 32.5 \mu \mathrm{m}$, and $71.42 \mu \mathrm{m}$. We then calculated $\chi^{2}$ and the $\chi^{2}$ probability distribution, where the fit is done to the observed five photometric points in Table 1 with two free parameters $\left(R_{\min }\right.$ and $\left.M_{\text {dust }}\right)$. The colors indicate the goodness of the fit. Of the wide range of models explored, only the two sets of models shown can fit the observations. Figure 5 (bottom) shows the parameter space $\left(R_{\min }\right.$ and $\left.M_{\text {dust }}\right)$ of the models in the top two panels. The SED modeling is degenerate, but compared to HD 128311, the case of HD 202206 is better constrained because of the detection of an excess starting near $25 \mu \mathrm{m}$. For the models that assume a single grain size, the best fits are given by $R_{\text {out }}=50 \mathrm{AU}$ and $10 \mathrm{AU} \leqslant R_{\text {in }} \leqslant 20 \mathrm{AU}$; and $R_{\text {out }}=100 \mathrm{AU}$ and $6 \mathrm{AU} \leqslant R_{\text {in }} \leqslant 10 \mathrm{AU}$. For this case, we can rule out the presence of a small $10 \mathrm{AU}$ disk. We can also rule out the models that assume a distribution of grain sizes with a power-law index of $-3.5, b_{\min }=b_{\text {blow }}$, and $b_{\max }=10 \mu \mathrm{m}$. The latter might not be surprising because collisional disk models by several authors (e.g., Krivov et al. 2006; Thébault \& Augereau 2007; Müller et al. 2010, Krivov 2010) conclude that the size distribution shows substantial deviations from a power law near the blow-out size regime, suggesting that $b_{\text {min }}$ would need to be two or three times larger than $b_{\text {blow }}$, and that the power-law 
(a) Single grain size: $10 \mu \mathrm{m}$ (b) Single grain size: $10 \mu \mathrm{m}$

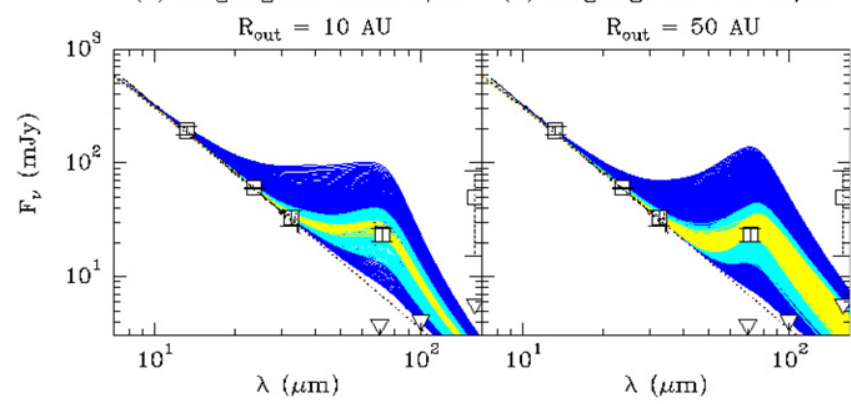

(d) Grain size distribution:

(c) Single grain size: $10 \mu \mathrm{m} \quad b_{\min }=0.17 \mu \mathrm{m} ; b_{\max }=10 \mu \mathrm{m}$

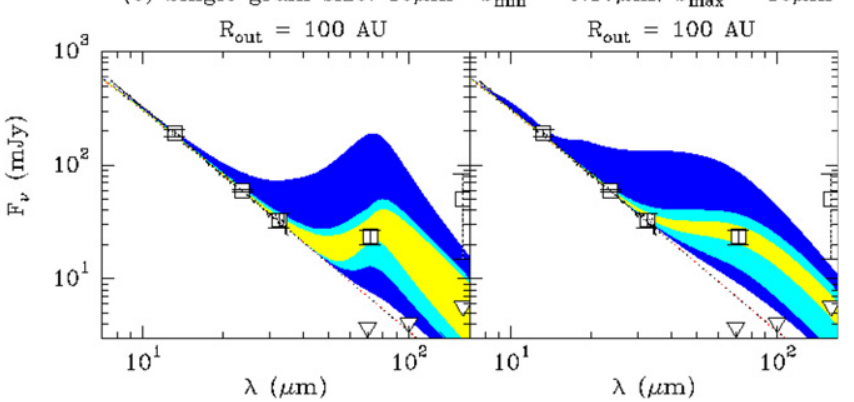

Figure 3. Observed and modeled SEDs for HD 128311. The dotted line is the stellar photosphere. The Spitzer photometric points (MIPS and synthetic photometry from IRS) are represented by squares with $1 \sigma$ error bars. The continuous lines correspond to the modeled SEDs and include the emission from the photosphere and from a disk of dust composed of particles with optical properties typical of astronomical silicates. Panels (a)-(c) correspond to $10 \mu \mathrm{m}$ size grains. Panel (d) corresponds to a grain size distribution given by $n(b) \propto$ $b^{-3.5}$ and a maximum grain radius of $b_{\max }=10 \mu \mathrm{m}$; regarding the minimum grain radius, we adopt the arbitrary value of $b_{\min }=0.17 \mu \mathrm{m}$ because the low luminosity of HD 128311 results in the absence of a blow-out size. The dust disk has a mass $M_{\text {dust }}$ and extends from $R_{\text {in }}$ to $R_{\text {out }}$ with a constant surface density. $R_{\text {in }}$ and $M_{\text {dust }}$ are the free parameters; $R_{\text {out }}$ is kept fixed at $10 \mathrm{AU}, 50 \mathrm{AU}$, and $100 \mathrm{AU}$ (indicated at the top of each panel). The models in yellow are those with a $\chi^{2}$ probability $P\left(\chi^{2} \mid v\right)<0.683$; light blue for $P\left(\chi^{2} \mid v\right)>0.683$, i.e., models that can be excluded with $1 \sigma$ certainty; and dark blue for $P\left(\chi^{2} \mid v\right)>0.9973$, i.e., models that are excluded with $3 \sigma$ certainty. The triangles correspond to the $5 \sigma / 1 \mathrm{hr}$ sensitivity limits for Herschel/PACS: $3.75 \mathrm{mJy}(60 \mu \mathrm{m}-85 \mu \mathrm{m})$, $4.1 \mathrm{mJy}(85 \mu \mathrm{m}-130 \mu \mathrm{m})$, and $5.75 \mathrm{mJy}(130 \mu \mathrm{m}-210 \mu \mathrm{m})$.

index may be smaller than 3.5 , to mimic a secondary maximum seen in the size distribution at hundreds of microns.

\subsection{HD 82943}

Because the IRS spectrum of HD 82943 indicates there is no dust excess at $\lambda<22 \mu \mathrm{m}$, we used the IRS synthetic photometry flux calculated between 15 and $19 \mu \mathrm{m}$ to pin down the photosphere. For simplicity, the stellar emission was modeled as a blackbody with $T_{\text {eff }}=5989 \mathrm{~K}, L_{*}=1.25 L_{\odot}$, and a distance of $27.46 \mathrm{pc}$.

For a stellar mass of $M_{*}=1.15 M_{\odot}$, a grain density $\rho=$ $2.5 \mathrm{~g} \mathrm{~cm}^{-3}$ and adopting the optical constants of astronomical silicates, the blow-out size is $b_{\text {blow }}=0.6 \mu \mathrm{m}$.

Figure 6 (top) shows the SED models overplotted on the Spitzer observations. For each model, we calculated the synthetic photometry using the MIPS and adopted IRS filter profiles at effective wavelengths of $13.2 \mu \mathrm{m}, 19 \mu \mathrm{m}, 23.68 \mu \mathrm{m}$, $32.5 \mu \mathrm{m}$, and $71.42 \mu \mathrm{m}$. We then calculated $\chi^{2}$ and the $\chi^{2}$ probability distribution, where the fit is done to the observed five photometric points in Table 1 with two free parameters $\left(R_{\min }, M_{\text {dust }}\right)$. The colors indicate the goodness of the fit. Figure 6 (bottom) shows the parameter space of the models in the top panels. (a) Single grain size $=10 \mu \mathrm{m}$ $\mathrm{R}_{\text {out }}=10 \mathrm{AU}$

(b) Single grain size $=10 \mu \mathrm{m}$ $\mathrm{R}_{\text {out }}=50 \mathrm{AU}$
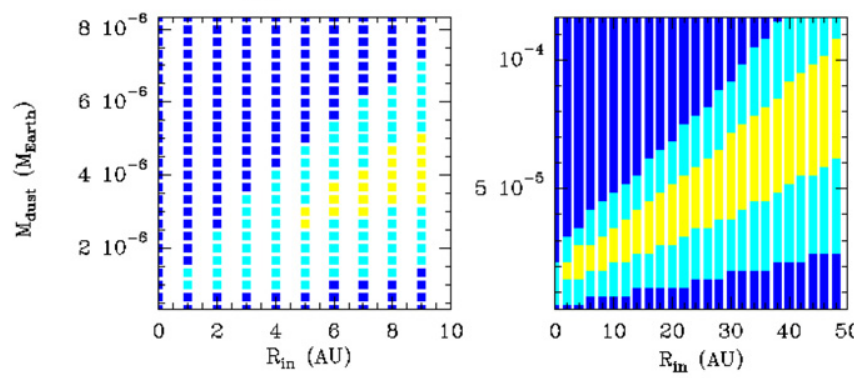

(c) Single grain size $=10 \mu \mathrm{m}$ $\mathrm{R}_{\text {out }}=100 \mathrm{AU}$

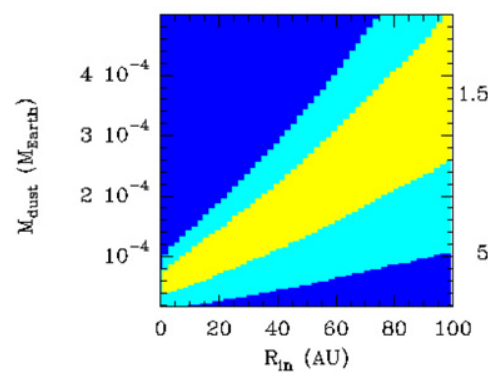

(d) Grain size distribution: $\mathrm{b}_{\min }=0.17 \mu \mathrm{m} ; \mathrm{b}_{\max }=10 \mu \mathrm{m}$

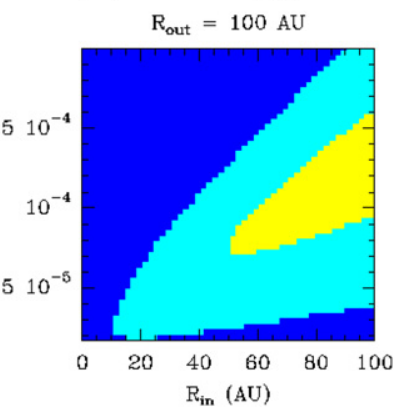

Figure 4. Parameter space of the modeled SEDs in Figure 3. Each point of these two-dimensional grids represents a modeled SED from Figure 3, where $R_{\text {in }}$ and $M_{\text {dust }}$ are the two free parameters. The models in yellow are those with a $\chi^{2}$ probability $P\left(\chi^{2} \mid v\right)<0.683$; light blue for $P\left(\chi^{2} \mid v\right)>0.683$, i.e., models that can be excluded with $1 \sigma$ certainty; and dark blue for $P\left(\chi^{2} \mid v\right)>0.9973$, i.e., models that are excluded with $3 \sigma$ certainty.

(a) Single grain size: $10 \mu \mathrm{m}$ (b) Single grain size: $10 \mu \mathrm{m}$

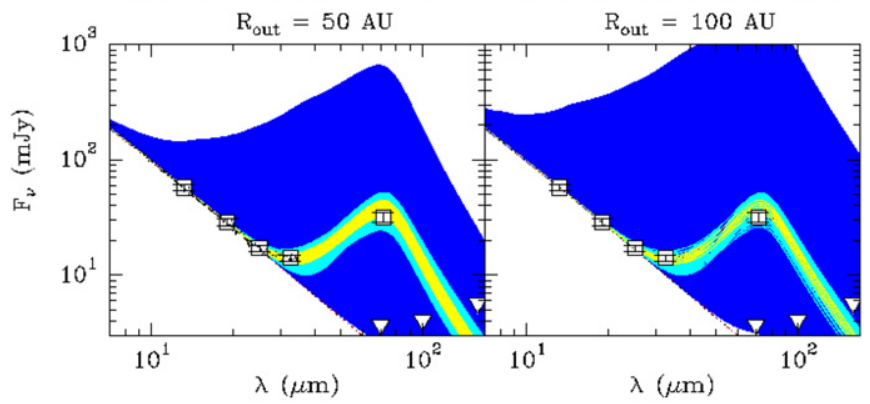

(a) Single grain size: $10 \mu \mathrm{m}$

(b) Single grain size: $10 \mu \mathrm{m}$
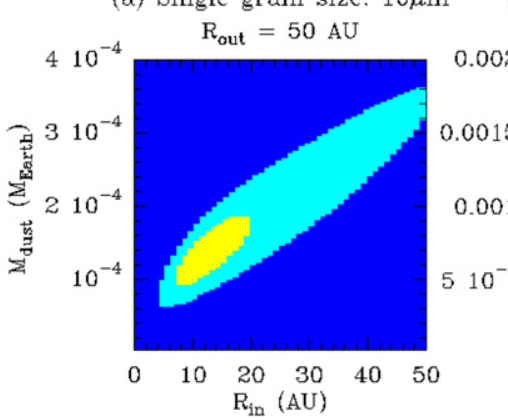
$\mathrm{R}_{\text {out }}=100 \mathrm{AU}$

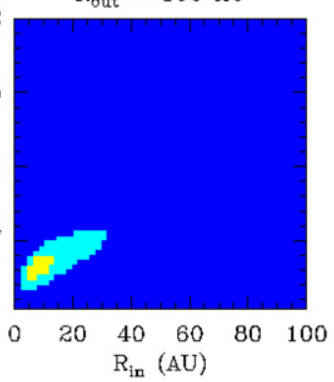

Figure 5. Same as Figures 3 and 4 but for HD 202206. Of the range of models explored, only the two sets of models shown in the panels could fit the observations.

For the models that assume a single grain radius of $10 \mu \mathrm{m}$, the best SED fits are given by $R_{\text {out }}=50 \mathrm{AU}$ and $16 \mathrm{AU} \leqslant R_{\text {in }}$ $\leqslant 44 \mathrm{AU}$; and $R_{\text {out }}=100 \mathrm{AU}$ and $12 \mathrm{AU} \leqslant R_{\text {in }} \leqslant 26 \mathrm{AU}$. For 
(a) Single grain size: $10 \mu \mathrm{m}$ (b) Single grain size: $10 \mu \mathrm{m}$

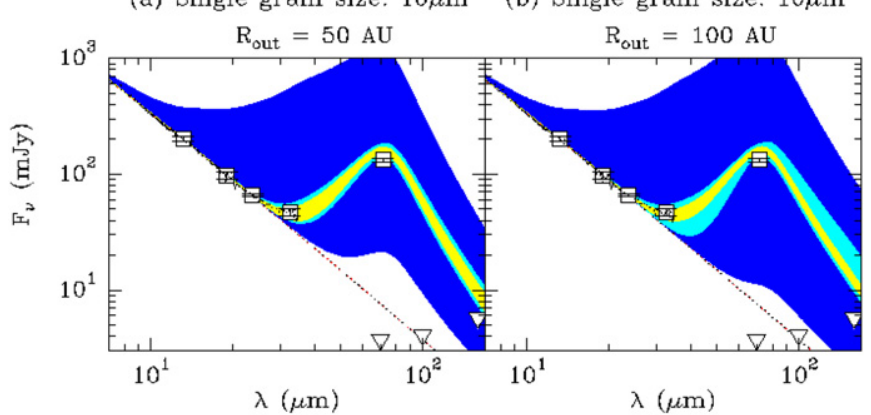

(a) Single grain size: $10 \mu \mathrm{m}$ $\mathrm{R}_{\text {out }}=50 \mathrm{AU}$

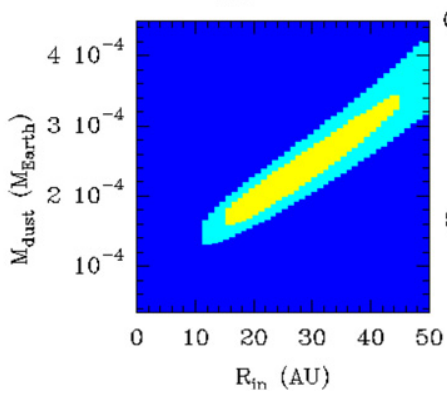

(b) Single grain size: $10 \mu \mathrm{m}$ $\mathrm{R}_{\text {out }}=100 \mathrm{AU}$

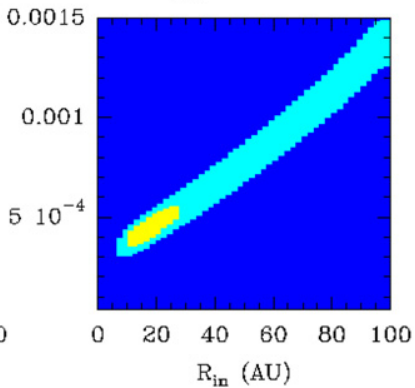

Figure 6. Same as Figures 3 and 4 but for HD 82943. Of the range of models explored, only the two sets of models shown in the panels could fit the observations.

this case, we can rule out the presence of a small 10 AU disk. As for HD 202206, we can also rule out models that assume a grain size distribution with a power-law index of $-3.5, b_{\min }=$ $b_{\text {blow }}$, and $b_{\max }=10 \mu \mathrm{m}$.

\subsection{HR 8799}

The Spitzer observations of HR 8799 and the analysis of its SED and spatially resolved images were presented in $\mathrm{Su}$ et al. (2009). Here, we summarize the main results. The stellar emission is best fitted by a Kurucz model with $T_{\text {eff }}=7500 \mathrm{~K}$, $R_{*}=1.4 R_{\odot}, \log (g)=4.5$, and sub-solar abundances, with a resulting $L_{*}=5.7 L_{\odot}$ (consistent with a young age). The stellar mass is $M_{*}=1.5 M_{\odot}$. As in Sections 3.1 and 3.2, Su et al. (2009) assumed spherical grains with optical properties characteristic of astronomical silicates, a bulk density of $2.5 \mathrm{~g} \mathrm{~cm}^{-3}$, and a size distribution following $n(b) \propto b^{-3.5}$; the blow-out size for HR 8799 is $2 \mu \mathrm{m}$. Su et al. (2009) found that the stellarsubtracted images and SED (after applying color corrections) can be best fitted by the following multi-component disk model:

1. Unresolved warm disk with a characteristic temperature of $\sim 150 \mathrm{~K}$. This component was modeled with a flat surface density, $\Sigma \sim r^{0}$. The maximum inner radius is $R_{\text {in }}=6 \mathrm{AU}$ (based on temperature arguments) and the outer radius is $R_{\text {out }}=15 \mathrm{AU}$. The grain sizes range from 1 to $4.5 \mu \mathrm{m}$ (with the spectral shape between 10 and $20 \mu \mathrm{m}$ requiring the presence of $\mu \mathrm{m}$-sized grains and the absence of submicron grains). If we were to assume all the grains are bound ( $>2 \mu \mathrm{m}$ ), the outer radius of this warm component would be $R_{\text {out }} \sim 10$ AU (instead of 15 AU). The estimated dust mass is $1.1 \times 10^{-6} M_{\oplus}$. This is a lower limit because it is not possible to constrain the mass contributed by larger grains as there are no long wavelength detections of this warm component. This component dominates the emission at IRS wavelengths and the unresolved emission at $24 \mu \mathrm{m}$ (it is too warm to contribute to the unresolved emission at $70 \mu \mathrm{m})$. The upper limit to the dust mass $<6 \mathrm{AU}$ is $2.3 \cdot 10^{-7} M_{\oplus}$ (assuming $10 \mu \mathrm{m}$ grains).

2. Unresolved cold disk with a characteristic temperature of $\sim 45 \mathrm{~K}$. This component was also modeled with a flat surface density, $\Sigma \sim r^{0}$. The inner radius, $R_{\text {in }}=$ $90 \mathrm{AU}$, is not constrained from imaging but from the SED: derived from the characteristic temperature of the cold component assuming blackbody grains $10 \mu \mathrm{m}$ in size. The sharp inflection between the IRS and the MIPSSED spectra indicates that there is a sharp inner edge of the cold dust component (i.e., there is very little dust located between the warm and the cold dust components). The outer radius is set to $R_{\text {out }}=300 \mathrm{AU}$, but is less well constrained because of the lack of spatially resolved submillimeter observations; the value of $300 \mathrm{AU}$ is chosen so that the $\mu \mathrm{m}$-sized grains in the extended halo (discussed next), assumed to originate from this cold component, are not too warm so as to make the emission at $25-35 \mu \mathrm{m}$ inconsistent with the observations. The grain sizes range from 10 to $1000 \mu \mathrm{m}$. Large grains are assumed to exist because this is the location of the collisional cascade that populates the extended halo discussed below. The estimated dust mass of this component is $0.12 M_{\oplus}$. This component is needed to fit the $\mathrm{SED}>30 \mu \mathrm{m}$. It dominates the unresolved emission at $70 \mu \mathrm{m}$ and constitutes $<9 \%$ of the total (unresolved+resolved) emission at $24 \mu \mathrm{m}$. This component also accounts for the unresolved submillimeter emission in Williams \& Andrews (2006).

3. Extended halo. At $24 \mu \mathrm{m}$, the cold component discussed above would be barely resolved, and a $70 \mu \mathrm{m}$ it would be unresolved, therefore, it is inferred that most of the resolved $24 \mu \mathrm{m}$ and $70 \mu \mathrm{m}$ emission comes from an extended halo component. It is modeled with a surface density, $\Sigma \sim$ $r^{-1}$, characteristic of an unbound disk. The limited spatial resolution at $70 \mu \mathrm{m}$ does not allow determination of the inner radius; it is placed at $R_{\mathrm{in}}=300 \mathrm{AU}$ because it is assumed the grains in the halo originate in a collisional cascade in the unresolved cold disk. $R_{\text {out }}$ is set to $1000 \mathrm{AU}$ because the disk can be traced even beyond this distance (the best-fit outer radius ranges from $900 \mathrm{AU}$ to $1800 \mathrm{AU}$ ). The grain sizes range from 1 to $10 \mu \mathrm{m}$ (grains larger than the blow-out size are included to account for the effects of porosity on the response of the grains to radiation pressure). Assuming a grain size distribution following $n(b) \propto b^{-3.5}$ and a maximum grain size of $10 \mu \mathrm{m}$, the ratio of the 70 and $24 \mu \mathrm{m}$ fluxes of the extended component is consistent with a minimum grain size of $\lesssim 2 \mu \mathrm{m}$ (similar to the blowout size for HR 8799). The estimated dust mass of this component is $1.9 \times 10^{-2} M_{\oplus}$.

In Section 5.4, we discuss the above results from $\mathrm{Su}$ et al. $(2009)^{8}$ in the context of the dynamical simulations in

\footnotetext{
8 Reidemeister et al. (2009) also carried out an analysis of the SED of HR 8799. They assume a flat surface density distribution $\left(\Sigma \sim r^{0}\right)$, astronomical silicate composition, and particle size distribution of $n(b) \propto b^{-3.5}$ with $b_{\min }=5 \mu \mathrm{m}$ and $b_{\max }=1000 \mu \mathrm{m}$. They inferred the presence of a warm component with $R_{\text {out }}=10-15 \mathrm{AU}$, and a cold component with $R_{\text {in }}=$ 75-120 AU and $R_{\text {out }}=125-170$ AU. We will take this result with caution because: (1) this fit does not include the deep IRS and spatially resolved MIPS observations (published after Reidemeister et al. 2009 paper), (2) they noted that the calibration of the (shallower) IRS data used is uncertain, and (3) we noted that their color corrections for the IRAS observations were done for a characteristic temperature of $5000 \mathrm{~K}$, instead of the much smaller dust temperature (that would lead to smaller correction factors).
} 

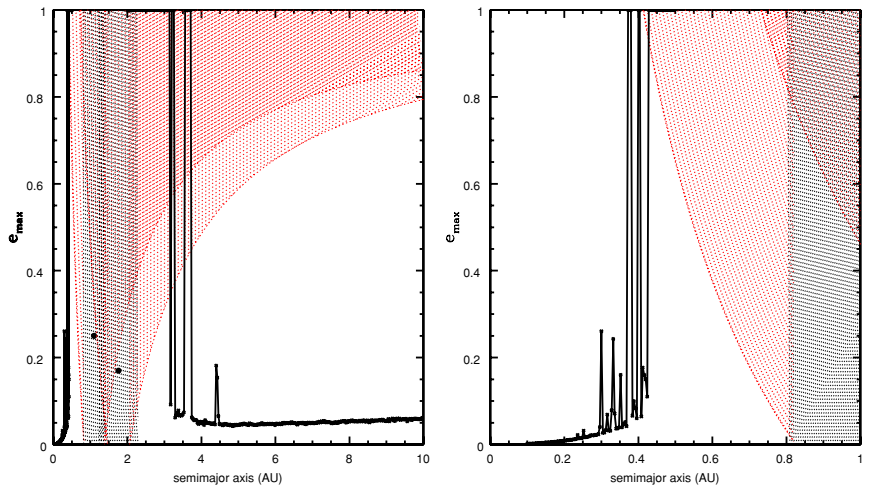

Figure 7. Allowed parameter space for the planetesimals' orbital elements. Shaded area: regions where test particle orbits are unstable due to planet-crossing (red) or overlapping first-order mean motion resonances (gray). The large black dots represent the two planets. The connected crosses are the maximum eccentricity attained by test particles on initially circular orbits, resulting from a numerical integration lasting $1 \mathrm{Myr}$. A zoom in to the region interior to the planets is shown in the right panel. The zone inside $\sim 0.3 \mathrm{AU}$ and the zone beyond $\sim 4 \mathrm{AU}$ appears stable for potential locations of planetesimal belts.

Section 4.4; these simulations consider the five possible planetary configurations listed in Table 2 and described in Section 2.4.

\section{DYNAMICAL MODELING}

\section{1. $H D 128311$}

Using numerical simulations, we have studied the effect of short- and long-term planetary perturbations on the orbital stability of the dust-producing planetesimals. Notice that with the orbital elements adopted in this paper for HD 128311 (Models A1 and A2 in Table 2), the planets are just barely outside their own planet-crossing zones. Because of the proximity of the planets' orbits to each other and to the 2:1 resonance, the analytical theory for the secular perturbations cannot be applied (as was done for HD 38529 in Moro-Martín et al. 2007b), and therefore we study the stability of the orbits using numerical integrations. Figure 7 corresponds to fit $\mathrm{A} 1$ and shows the maximum eccentricity attained by test particles on initially circular orbits, resulting from gravitational perturbations by the two planets for an integration time of 1 Myr. We can exclude the presence of planetesimals in the regions shaded with black dots because those orbits are chaotic due to overlapping firstorder MMRs (Wisdom 1980); similarly, the regions shaded with red dots are not expected to have stable planetesimals because they are planet-crossing eccentric orbits. The stability of the potential dust-producing planetesimal belt was also studied for the orbital solution in fit A2 (Figure 8). For this simulation, we used 500 particles uniformly spaced between $0.5 \mathrm{AU}$ and $100 \mathrm{AU}$, on initially circular orbits co-planar with the planets, with angular elements chosen randomly between 0 and $2 \pi$. Particles were removed if they approached the star closer than $0.5 \mathrm{AU}$, or approached a planet closer than its Hill radius. The orbits were integrated for $\sim 100 \mathrm{Myr}$ using the multiple time step symplectic method skeel-SyMBA (Duncan et al. 1998). The evolution of the planets' semimajor axes and eccentricities is shown in Figure 9. The potential locations of planetesimal belts are the regions where the orbits are stable and where the maximum eccentricity is low $(\lesssim 0.3)$, ensuring long planetesimal lifetimes. Figures 7 and 8 show that there are two such regions: (1) interior to $\sim 0.3 \mathrm{AU}$ and (2) exterior to $\sim 4 \mathrm{AU}$ (for $a>4 \mathrm{AU}$ the maximum eccentricity always remains below 0.05 ).
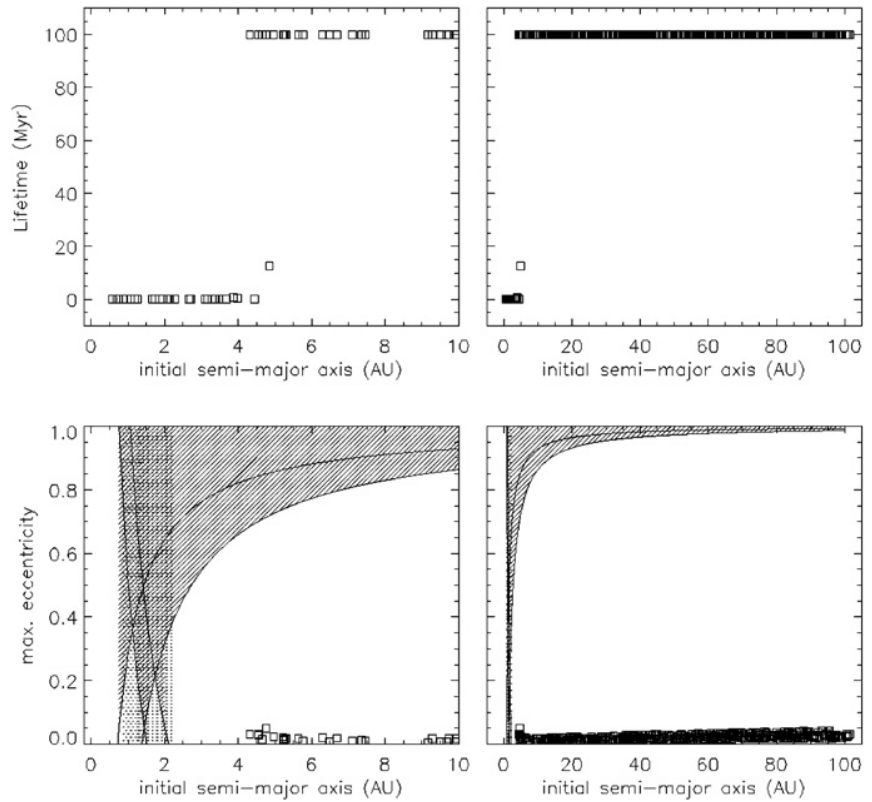

Figure 8. Results from the dynamical simulation of 500 test particles in the HD 128311 planetary system (fit A2). A zoom in to the inner system is shown in the left panels. The numerical integration lasted 100 Myr. Top: test particles' lifetimes. Bottom: allowed parameter space for the planetesimals' orbital elements, where the shaded areas indicate regions where the test particles' orbits are unstable due to planet-crossing (striped area) or overlapping first-order mean motion resonances (dotted area); the squared symbols show the maximum eccentricity attained by test particles on initially circular orbits.

\subsection{HD 202206}

The stability of the potential dust-producing planetesimals was studied using numerical simulations of test particles with the planetary parameters listed in Table 2 (fit B1). The simulations were done for 500 particles uniformly spaced between $0.5 \mathrm{AU}$ and $100 \mathrm{AU}$, with an integration time of $100 \mathrm{Myr}$. The evolution of the planets' semimajor axes and eccentricities during that time span is shown in Figure 9. The results shown in Figure 10 indicate that the test particle's orbits are stable beyond $\sim 6 \mathrm{AU}$, and that their maximum eccentricity remains $\lesssim 0.3$ for all semimajor axis; this indicates that the dust-producing planetesimals are located beyond $6 \mathrm{AU}$.

\subsection{HD 82943}

We have studied numerically the stability of the potential dustproducing planetesimals in the three planetary configurations discussed in Section 2.3 (listed in Table 2). The simulations were done for 500 particles uniformly spaced between $0.5 \mathrm{AU}$ and $100 \mathrm{AU}$, with an integration time of $\gtrsim 70 \mathrm{Myr}$. The evolution of the planets' semimajor axes and eccentricities is shown in Figure 9. The results in Figures 11-13 indicate that, in the three planetary configurations considered, the test particle orbits are stable beyond $\sim 3 \mathrm{AU}$, with maximum eccentricities always $<0.1$. Long-lived, dust-producing planetesimals could therefore be located anywhere beyond $3 \mathrm{AU}$.

\section{4. $H R 8799$}

We have studied the stability of the potential dust-producing planetesimals using numerical simulations of test particles in the five planetary configurations discussed in Section 2.4 and listed in Table 2. The simulations consisted of 1500 test particles: 500 particles uniformly spaced between $2 \mathrm{AU}$ and the semimajor 

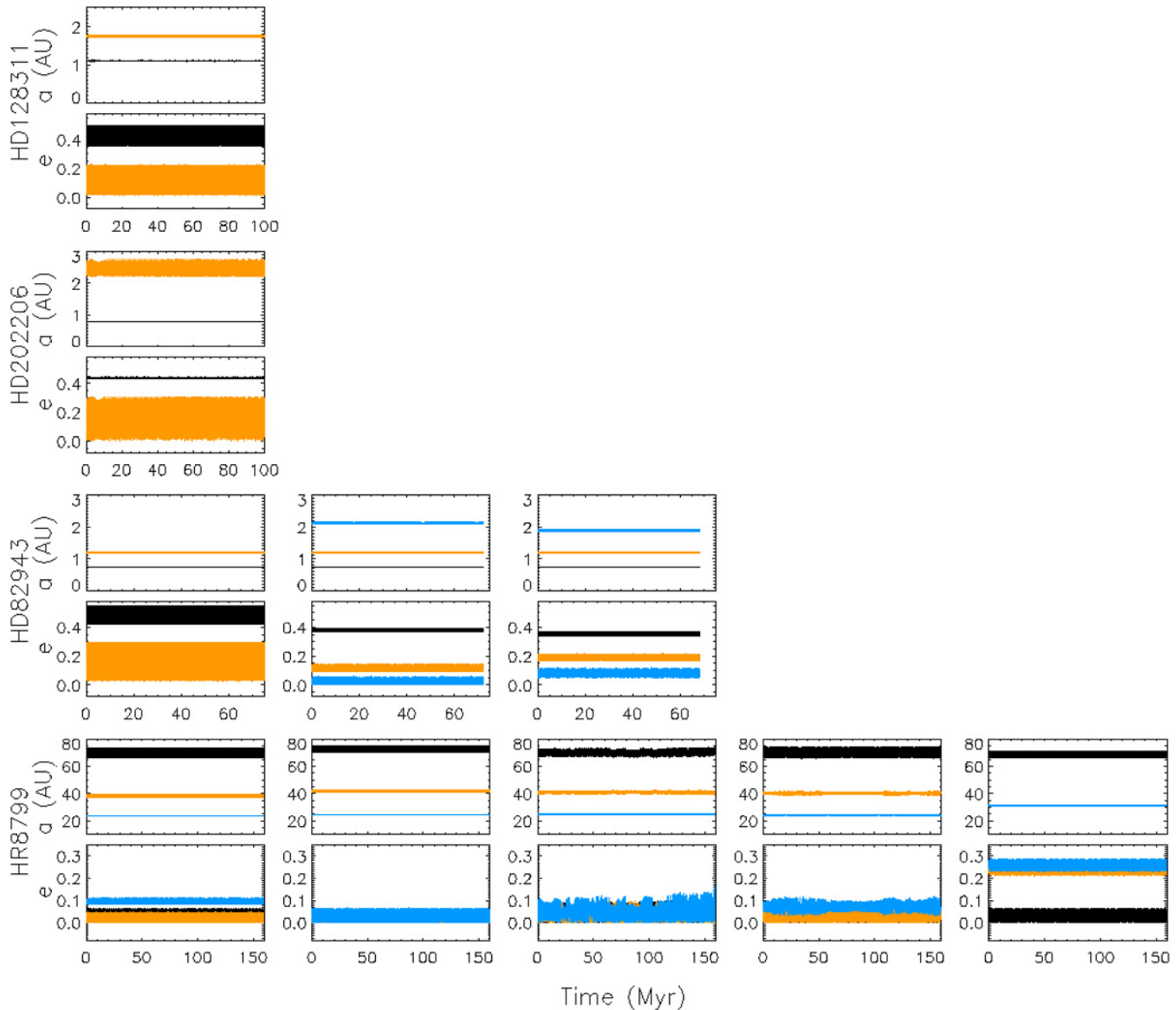

Figure 9. Long-term evolution of the planets' semimajor axes and eccentricities for the planetary configurations in Table 2. From top to bottom, left to right, the panels correspond to fits A2, B1, C1-C3, and D1-D5, for HD 128311, HD 202206, HD 82943, and HR 8799, respectively. The models were run for 100 Myr (HD 128311), $100 \mathrm{Myr}$ (HD 202206), ¿70 Myr (HD 82943), and $160 \mathrm{Myr}$ (HR 8799). The colors correspond to planet b (black), c (orange), and d (blue).
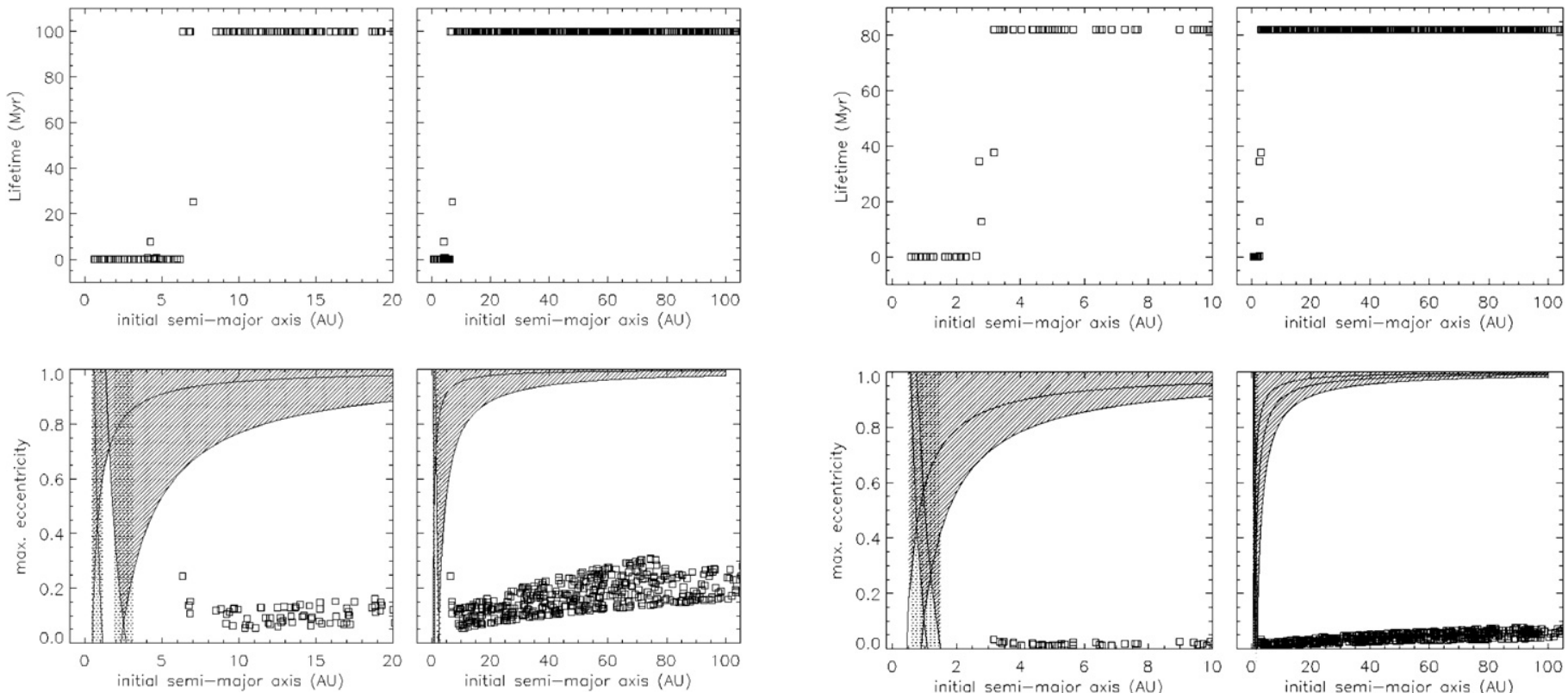

Figure 10. Same as Figure 8 for 500 test particles in the HD 202206 planetary system (fit B1); the numerical integration lasted $100 \mathrm{Myr}$.
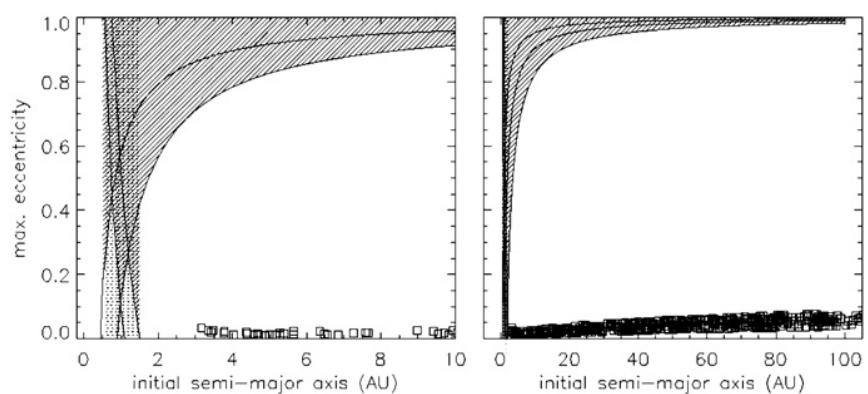

Figure 11. Same as Figure 8 for 500 test particles in the HD 82943 planetary system (fit C1); the numerical integration lasted $\sim 82 \mathrm{Myr}$. 

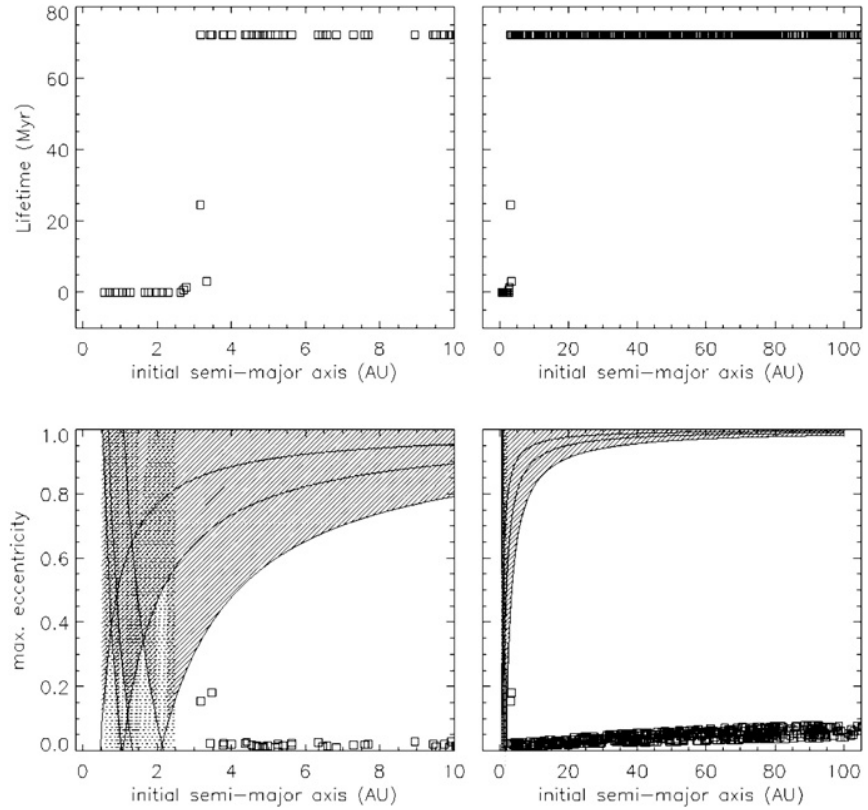

Figure 12. Same as Figure 8 but for fit C2 of HD 82943; the numerical integration lasted $\sim 72$ Myr.
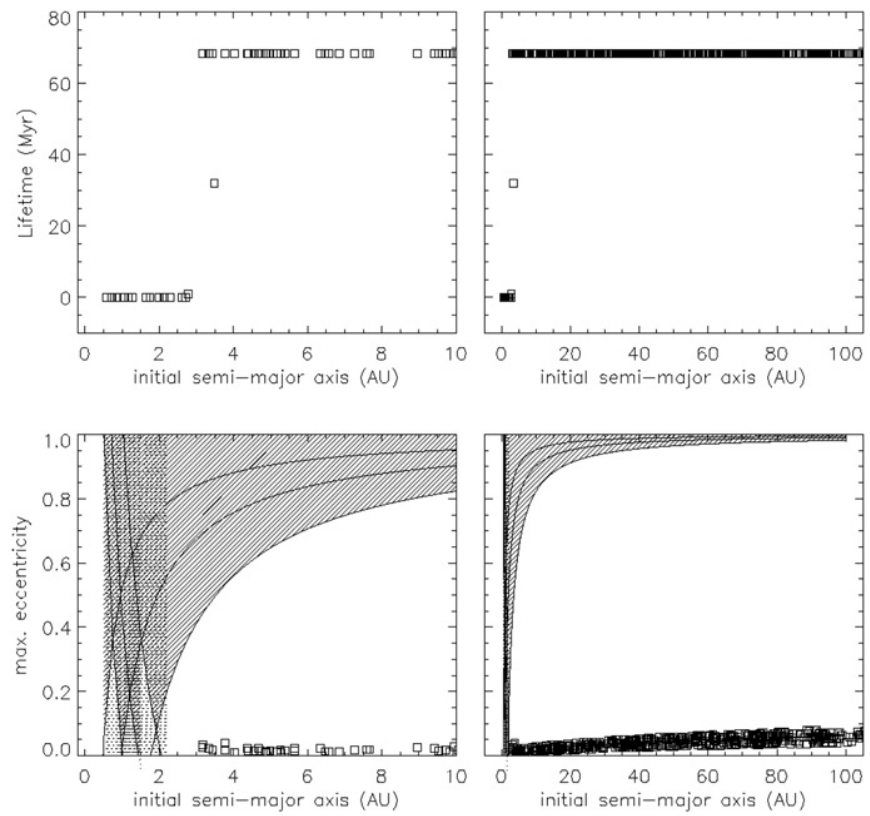

Figure 13. Same as Figure 8 but for fit C3 of HD 82943; the numerical integration lasted $\sim 68 \mathrm{Myr}$.

axis of the innermost planet, 500 particles located between the innermost and outermost planet, and 500 particles uniformly spaced between the semimajor axis of the outermost planet and 300 AU. This latter value is taken from the results in the SED analysis in Su et al. (2009) summarized in Section 3.4. We assumed that the planets and the dust-producing planetesimals formed out of a thin disk and are co-planar. Particles were removed if they approached the star closer than 2 AU, or approached a planet closer than its Hill radius. The orbits were integrated for $160 \mathrm{Myr}$ (the upper limit to the age of HR 8799 as estimated by Marois et al. 2008). The semimajor axes and eccentricities of the planet orbits do not evolve significantly during that time span (see Figure 9). The results regarding the test particles are shown in Figures 14-18. For all the planetary
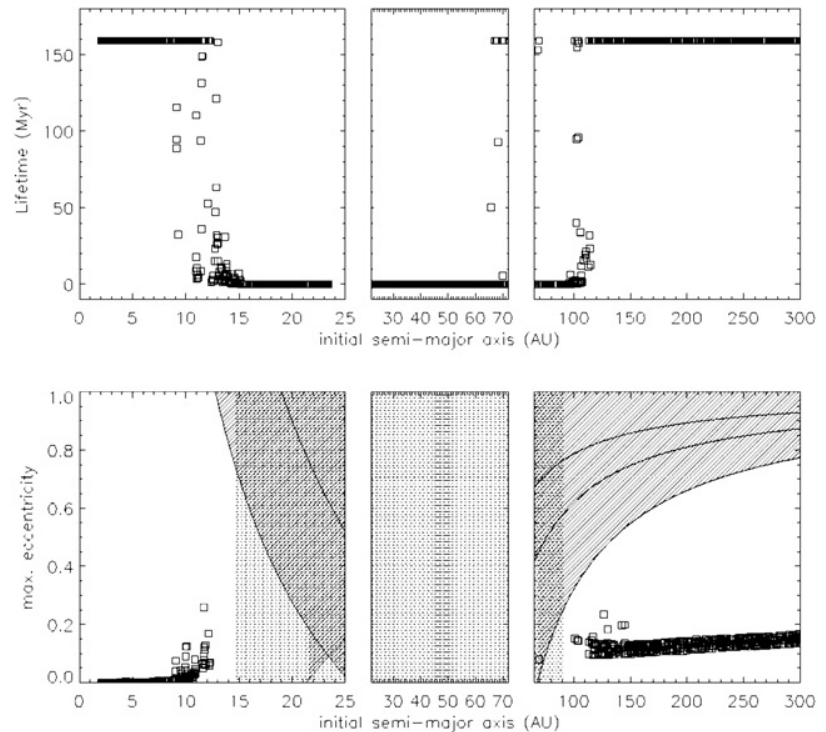

Figure 14. Same as Figure 8 for 1500 test particles in the HR 8799 planetary system (fit D1); the numerical integration lasted $160 \mathrm{Myr}$. The very slight rising trend of the maximum eccentricity at large semimajor axis seen in Figures 14-18 is a numerical artifact that arises because the test particle simulations for HR 8799 were carried out by skeel-SyMBA which gives orbital elements in stellar-centric osculating elements; this produces a spurious eccentricity in nearly circular orbits because the star is wobbling, and its wobble velocity becomes a larger and larger fraction of the orbital velocity of test particles at larger and larger distances. (This effect has been corrected in Figures 8 and 10-13, where the osculating Keplerian elements of the test particles are calculated in the barycentric frame.)

configurations considered, the region between the orbits of the planets is dynamically unstable. The test particles' orbits are stable in two regions: (1) $\lesssim 12$ AU for fits D1 and D2, and $\lesssim 10$ AU for fits D3, D4, D5 and (2) $\gtrsim 110$ AU for fits D1, D2, D5, and $\gtrsim 150$ AU for fits D3, D4. Regarding the maximum eccentricity of the test particles in the dynamically stable regions, Figures 14-18 show that $e_{\max } \lesssim 0.3$, even though for some fits there are peaks at $\sim 5 \mathrm{AU}$ and $\sim 170$ AU (due to secular resonances). Given the low maximum eccentricities, these dynamically stable regions could be possible locations of long-lived, dust-producing planetesimals (except at $\sim 5 \mathrm{AU}$ in fit D3 where the $e_{\max }$ reaches 0.4 and where the planetesimals' lifetimes might be short under collisional evolution).

\section{DISCUSSION: POTENTIAL LOCATION OF THE DUST-PRODUCING PLANETESIMAL BELTS}

We now discuss the potential location of the dust-producing planetesimal belts in each for the four multiple-planet systems studied in this paper.

\subsection{HD 128311}

We favor models that assume a single grain radius of $10 \mu \mathrm{m}$ because they represent large blackbody grains in general, and there is no evidence of a significant population of small grains. With these models, the observed SED can be fitted by a dust disk with $R_{\text {out }}=10 \mathrm{AU}$ and $R_{\text {in }} \geqslant 5 \mathrm{AU}, R_{\text {out }}=50 \mathrm{AU}$ and $R_{\text {in }} \geqslant 2 \mathrm{AU}$, or $R_{\text {out }}=100 \mathrm{AU}$ and $R_{\text {in }} \geqslant 2 \mathrm{AU}$. The dynamical simulations help constrain further the possible location of the dust-producing planetesimals because the effects of the planets extend into the regions allowed by the SED models: they predict two stable niches where planetesimals could be long lived, 

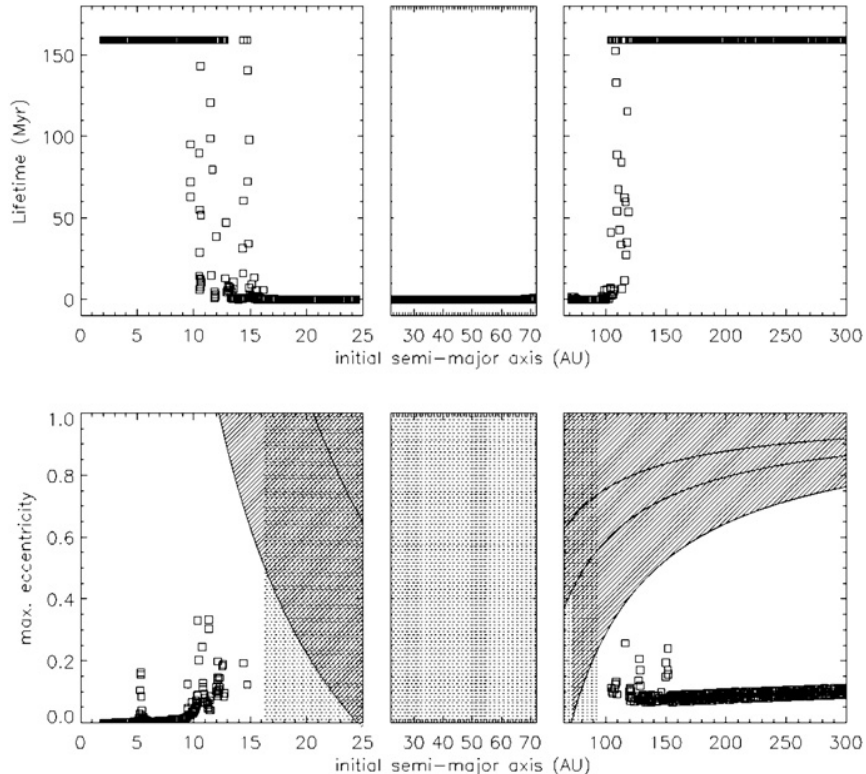

Figure 15. Same as Figure 14 but for fit D2 of HR 8799.
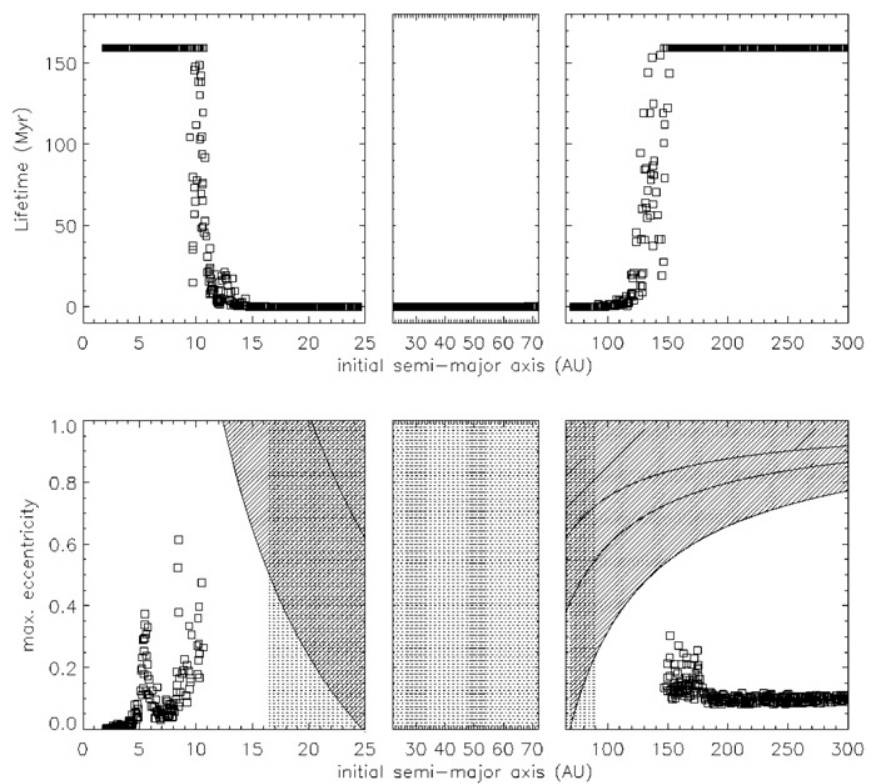

Figure 16. Same as Figure 14 but for fit D3 of HR 8799.

beyond $\sim 4 \mathrm{AU}$ and inside $\sim 0.3 \mathrm{AU}$. Regarding the latter, to account for the lack of excess emission at $\lambda<33 \mu \mathrm{m}$, the SED modeling excludes the presence of a significant population of dust-producing planetesimals in this region. To set tighter constraints to the location of the planetesimals in HD 128311 , there is the need to obtain spatially resolved images and/or accurate photometric points in the $33-70 \mu \mathrm{m}$ range and in the submillimeter. As seen in Figures 2 and 3, observations with the recently launched Herschel/PACS would be very valuable for this purpose.

\subsection{HD 202206}

The dynamical model indicates that planetesimal orbits are stable beyond $\sim 6 \mathrm{AU}$, and that their maximum eccentricity remains $\lesssim 0.1$ for all semimajor axes, i.e., that planetesimals could be long lived beyond 6 AU. This result agrees with the conclusions from the SED modeling. These models, which are well
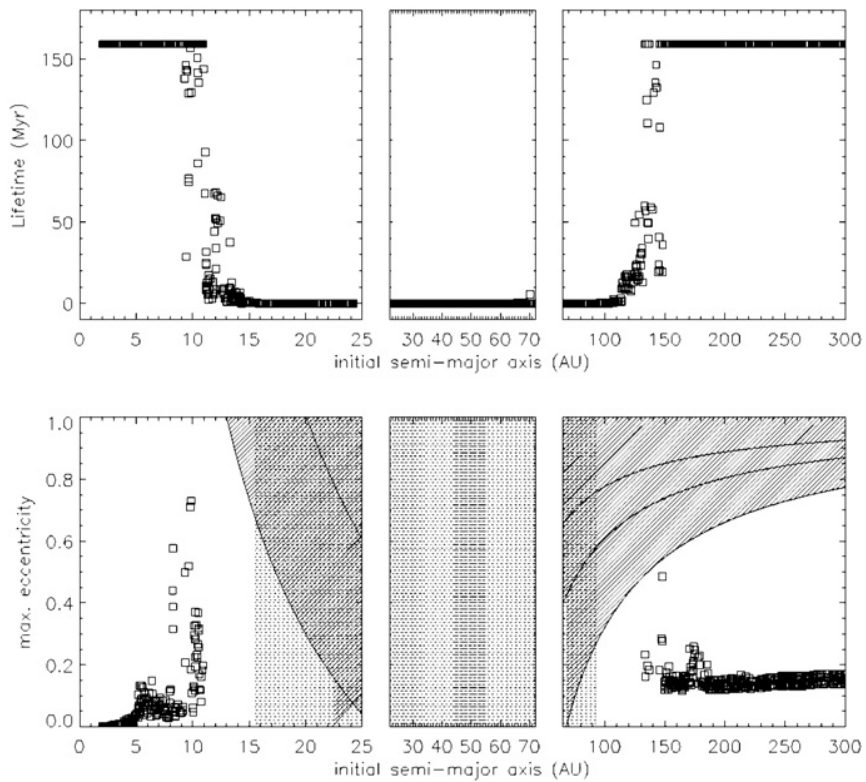

Figure 17. Same as Figure 14 but for fit D4 of HR 8799.
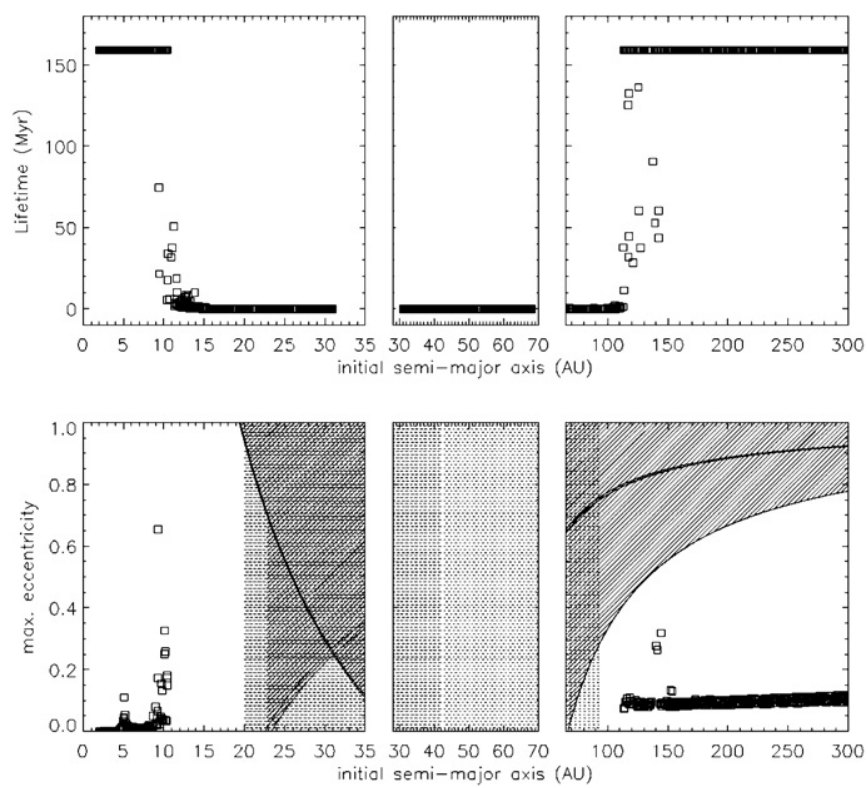

Figure 18. Same as Figure 14 but for fit D5 of HR 8799.

constrained because of the presence of a small excess beyond $25 \mu \mathrm{m}$, result in a relatively narrow range of planetesimal belts that can fit the observations, ranging from a $50 \mathrm{AU}$ disk with an inner cavity 10-20 AU in size, to a $100 \mathrm{AU}$ disk with an inner cavity 6-10 AU in size. We conclude that the gravitational perturbations of the detected planets might be responsible for the inner edge of the dust disk.

\subsection{HD 82943}

The observed SED, lacking emission at $\lambda<33 \mu \mathrm{m}$, can be fitted by a dust disk composed of single grains $10 \mu \mathrm{m}$ in size, with an inner cavity with $R_{\text {in }} \geqslant 16$ AU for a disk with $R_{\text {out }}=$ $50 \mathrm{AU}$, or $R_{\text {in }}=12-26 \mathrm{AU}$ for a disk with $R_{\text {out }}=100 \mathrm{AU}$ (a compact $10 \mathrm{AU}$ dust disk is excluded). Because the dynamical modeling of test particles in the three planetary configurations considered suggests that planetesimals could be stable and 

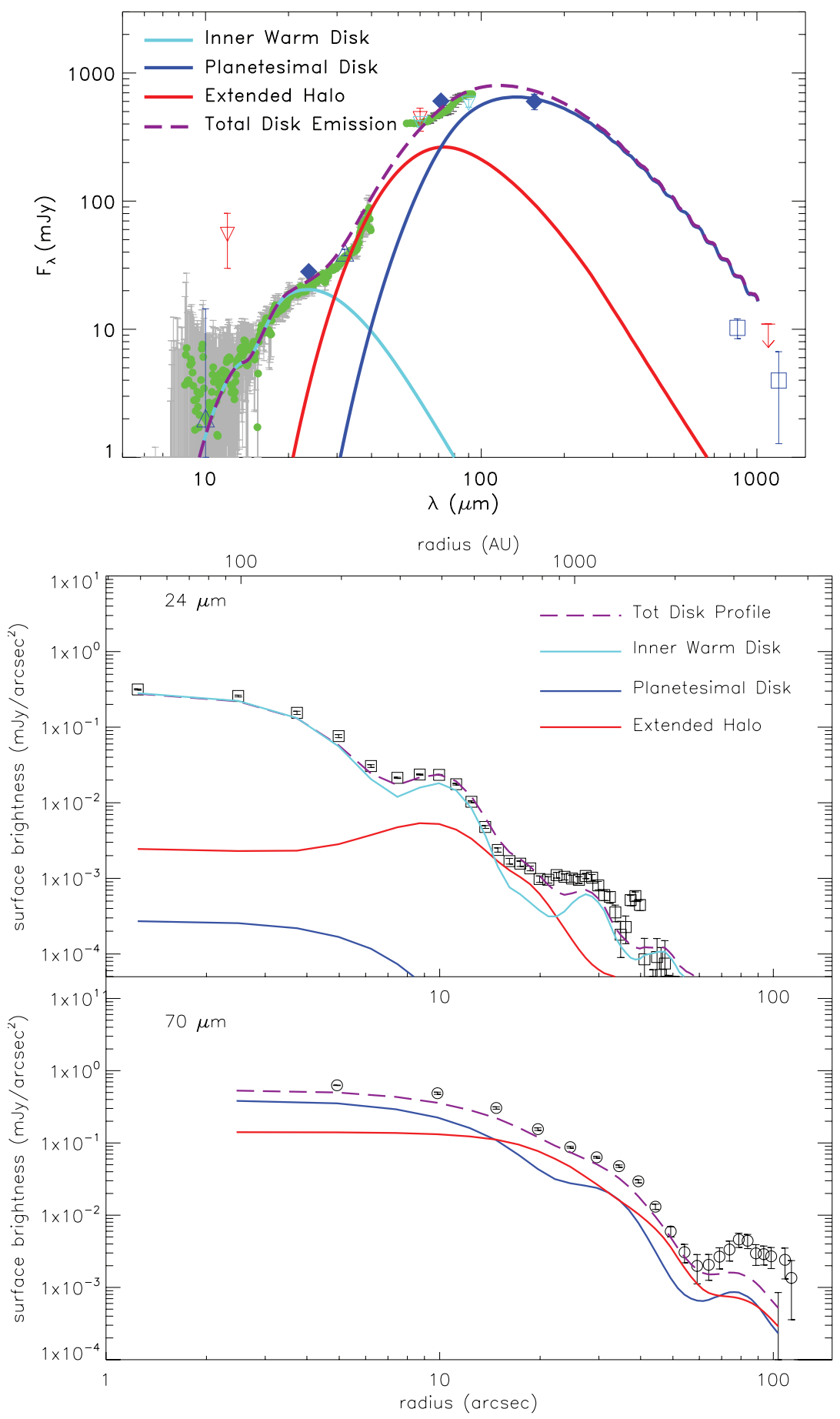

Figure 19. Same as Figure 9 in Su et al. (2009) but setting the inner edge of the cold component at $R_{\text {in }}=110$ AU instead of 90 AU. Compared to the model with $R_{\mathrm{in}}=90 \mathrm{AU}$, this fit gives a slightly worse $\chi^{2}$-value (by 20\%) but is still within the errors. (Top) Observed and modeled SED: the observations are represented as symbols with error bars and correspond to MIPS (dark blue diamonds; color-corrected), MIPS-SED (green), IRS (green), ISO (light blue triangles; color corrected), IRAS (red triangles; color corrected) and sub-millimeter (dark blue squares); regarding the latter, even though it appears that the model overestimates the flux in the sub-millimeter, both fluxes agree when taking into account the source extension (see Su et al. 2009 for details). (Bottom) Observed and modeled surface brightness profiles at $24 \mu \mathrm{m}$ and $70 \mu \mathrm{m}$; the observations are represented as open symbols with error bars. The parameters of the three disk components are as follows. Inner warm disk: $R_{\text {in }}=6 \mathrm{AU} . R_{\text {out }}=16 \mathrm{AU}$, a flat surface density $\left(\Sigma \sim r^{0}\right), M_{\text {dust }}=1.12 \cdot 10^{-6} M_{\oplus}$, and a grain size distribution with $b_{\min }=1.5 \mu \mathrm{m}, b_{\max }=4.5 \mu \mathrm{m}$ and $q=-3.5$. Outer cold disk: $R_{\mathrm{in}}=110 \mathrm{AU}, R_{\text {out }}=300 \mathrm{AU}$, a flat surface density $\left(\Sigma \sim r^{0}\right), M_{\text {dust }}=1.31 \cdot 10^{-1} M_{\oplus}$, and a grain size distribution with $b_{\text {min }}=10 \mu \mathrm{m}$, $b_{\max }=1000 \mu \mathrm{m}$ and $q=-3.5$. Extended halo: $R_{\text {in }}=300 \mathrm{AU}, R_{\text {out }}=1000 \mathrm{AU}$, a surface density given by $\Sigma \sim r^{-1}, M_{\text {dust }}=1.94 \cdot 10^{-2} M_{\oplus}$, and a grain size distribution with $b_{\min }=1 \mu \mathrm{m}, b_{\max }=10 \mu \mathrm{m}$ and $q=-3.5$. See Su et al. (2009) for details on the modeling.

long lived beyond $\sim 3 \mathrm{AU}$, we conclude that the gravitational perturbations from the planets (located within 2.1 AU of the star) do not extend far enough to have a significant effect to destabilize any debris system that is seen in the infrared excess emission.

\subsection{HR 8799}

For the five planetary configurations considered, the dynamical modeling of test particles suggests that planetesimals could be stable and long lived at semimajor axes $\lesssim 10-12$ AU and 
$\gtrsim 110-150 \mathrm{AU}$. This helps to further constrain the dust disk solutions based on the SED fitting by Su et al. (2009), who proposed: (1) the presence of an unresolved warm disk $15 \mathrm{AU}$ in size, if the grain sizes range from 1 to $4.5 \mu \mathrm{m}$, or a more compact $10 \mathrm{AU}$ disk, if all the grains are bound $(>2 \mu \mathrm{m})$ and (2) the presence of an unresolved cold disk with a sharp inner edge at $\sim 90$ AU. The dynamical models favor an inner dust disk component of bound grains and $R_{\text {out }} \sim 10 \mathrm{AU}$ and a colder component with $R_{\text {in }} \sim 110-150 \mathrm{AU}$ (rather than $\sim 90 \mathrm{AU}$ ). Regarding the cold component, we find there is no tension between $R_{\text {in }}$ derived from the dynamical models $(\sim 110-150 \mathrm{AU})$ and that derived from the analysis of the SED and the surface brightness radial profiles. Even though Su et al. (2009) favors a value of $R_{\text {in }} \sim 90$ AU, Figure 19 shows that the observations can also be fitted with $R_{\text {in }}=110 \mathrm{AU}$ (increasing the total dust mass) and $R_{\text {in }}=150$ AU (changing the minimum grain size from $10 \mu \mathrm{m}$ to $8 \mu \mathrm{m}$ ); this is not surprising because all these inner radii are smaller than the MIPS $70 \mu \mathrm{m}$ pixel size.

The presence of this outer planetesimal disk may help constrain planet formation scenarios that have been proposed for the HR 8799 system. One scenario is planet-planet scattering: in addition to the difficulty to result in a stable system with low eccentric planets (Dodson-Robinson et al. 2009), this model may not be able to account for the presence of the outer planetesimal disk. A second scenario is long-range outward migration in resonance (Crida et al. 2009): future high-resolution imaging of the cold component of the HR 8799 debris disk may be able to provide evidence of dust-producing planetesimals trapped in MMRs with the outermost planet, a signpost of outward planet migration.

Table 3 and Figure 1 summarize the possible planetplanetesimal configurations of HD 128311, HD 202206, HD 82943, and HR 8799, compared to that of the other three multi-planet systems known to harbor dust-HD 38529, HD 69830, and the Sun. In some cases, the SED allows for the presence of both narrow and wide belts. We favor the latter because the narrowest belts might only be justified in the presence of additional planetary perturbers for which we have no evidence so far. However, one should keep in mind the degeneracy: to set tighter constraints to the location of the planetesimals there is the need to obtain spatially resolved images and/or accurate photometric points in the $30-70 \mu \mathrm{m}$ range and in the submillimeter. Observations with Herschel/PACS and ALMA will be very valuable for this purpose.

\section{CONCLUSIONS}

In this paper, we have studied the possible planet-planetesimal configurations of four multi-planet systems, of which three are radial-velocity systems-HD 128311, HD 202206, and HD 82943-and one is a directly imaged system-HR 8799. We have quantified where the zone of influence lies of planets on the dust-producing planetesimals: for HR 8799 it extends to nearly $20 \mathrm{AU}$ from the orbit of the outermost planet, while for the three radial-velocity systems it extends to about $4 \mathrm{AU}$. A previous paper that studied HD 38529, another radial-velocity multi-planet system, found that the influence of the planets in this case extends out to $\sim 10 \mathrm{AU}$ (determining the inner edge of the disk), and becomes dominant again at a $\sim 55 \mathrm{AU}$ (due to a secular resonance that probably determines the outer edge of the dust disk; Moro-Martín et al. 2007b). We conclude that radial-velocity multi-planet systems generally have zones of influence within a few to ten $\mathrm{AU}$; more precise determinations will require individual modeling of a system. The influence can be extended much further through secular resonances and similar behavior.

For the three radial-velocity multi-planet systems studied in this paper, we have constructed fits to the SEDs of the debris disks. If we use astronomical silicates with a size distribution down to the blow-out size, the emitting regions of the disks must be so far from the star that they are well outside the zones of influence of the radial-velocity planets. This behavior depends critically on the optical properties of the grains (size distribution and optical constants of the grain material). If we were to adopt optical constants typical of an ice-silicate mixture instead of astronomical silicates, the dust may be located closer to the star. In all three cases, we find that single-size $10 \mu \mathrm{m}$ astronomical silicates reproduce well the observed SED, and could lie at the edge of the zone of influence of the planets and, therefore, the disk would be sculpted by them.

We thank Hal Levison for providing skeel-SyMBA for the dynamical simulations, Sebastian Wolf for providing DDS for the SED models, and Alexander Krivov for his careful reading of the manuscript and useful comments. This work is based on observations made with the Spitzer Space Telescope, which is operated by the Jet Propulsion Laboratory, managed for NASA by the California Institute of Technology. A.M.M. acknowledges funding from the Spanish MICINN (Ramón y Cajal Program and grants AYA2009-07304 and CONSOLIDER INGENIO 2010CSD2009-00038), the Michelson Fellowship, and the Spitzer archival grant 40412. She thanks the Isaac Newton Institute for Mathematical Sciences at Cambridge University for support. R.M. acknowledges support from grants by NSF (AST-0806828) and NASA (NNX08AQ65G).

\section{REFERENCES}

Backman, D., et al. 2009, ApJ, 690, 1522

Barnes, S. A. 2007, ApJ, 669, 1167

Beauge, C., Giuppone, C. A., Ferraz-Mello, S., \& Michtchenko, T. A. 2008, MNRAS, 385, 2151

Beichman, C. A., et al. 2005, ApJ, 622, 1160

Beichman, C. A., et al. 2006, ApJ, 639, 1166

Biller, B. A., et al. 2007, ApJS, 173, 143

Bryden, G., et al. 2009, ApJ, 705, 1226

Burns, J. A., Lamy, P. L., \& Soter, S. 1979, Icarus, 40, 1

Butler, R. P., et al. 2006, ApJ, 646, 505

Carpenter, J. M., et al. 2009, ApJS, 181, 197

Chauvin, G., et al. 2006, A\&A, 456, 1165

Chen, C. H., et al. 2009, ApJ, 701, 1367

Correia, A. C., M., et al. 2005, A\&A, 440, 751

Couetdic, J., et al. 2009, arXiv:0911.1963

Crida, A., Masset, F., \& Morbidelli, A. 2009, ApJ, 705, L148

Dermott, S. F., Kehoe, T. J., Durda, D. D., Grogan, K., \& Nesvorný, D. 2002, in Proc. Asteroids, Comets, Meteors, ed. B. Warmbein (ESA SP-500; Noordwijk: ESA), 319

Dodson-Robinson, S. E., Veras, D., Ford, E. B., \& Beichman, C. A. 2009, ApJ, 707, 79

Duncan, M. J., Levison, H. F., \& Lee, M. H. 1998, AJ, 116, 2067

Eggenberger, A., et al. 2007, A\&A, 474, 273

Fabrycky, D. C., \& Murray-Clay, R. A. 2010, ApJ, 710, 1408

Ferraz-Mello, S., Michtchenko, T. A., \& Beaugé, C. 2005, ApJ, 621, 473

Gáspár, A., Rieke, G. H., Su, K. Y. L., Balog, Z., Trilling, D., Muzzerole, J., Apai, D., \& Kelly, B. C. 2009, ApJ, 697, 1578

Gray, R. O., Corbally, C. J., Garrison, R. F., McFadden, M. T., Bubar, E. J., McGahee, C. E., O’Donoghue, A. A., \& Knox, E. R. 2006, AJ, 132, 161

Gray, R. O., Corbally, C. J., Garrison, R. F., McFadden, M. T., \& Robinson, P. E. 2003, AJ, 126, 2048

Goździewski, K., \& Konacki, M. 2006, ApJ, 647, 573

Goździewski, K., Konacki, M., \& Maciejewski, A. J. 2006, ApJ, 645, 688

Goździewski, K., \& Migaszewski, C. 2009, MNRAS, 397, L16

Hillenbrand, L. A., et al. 2008, ApJ, 677, 630 
Kalas, P., et al. 2010, BAAS, 41, 587

King, J. R., Villarreal, A. R., Soderblom, D. R., Gulliver, A. F., \& Adelman, S. J. 2003, AJ, 125, 1980

Krivov, A. V. 2010, Res. Astron. Astrophys., 10, 383

Krivov, A. V., Löhne, T., \& Sremčević, M. 2006, A\&A, 455, 509

Lafrenière, D., Marois, C., Doyon, R., \& Barman, T. 2009, ApJ, 694, L148

Lawler, S. M., et al. 2009, ApJ, 705, 89

Lee, M. H., Butler, R. P., Fischer, D. A., Marcy, G. W., \& Vogt, S. S. 2006, ApJ, 641,1178

Lisse, C. M., Beichman, C. A., Bryden, G., \& Wyatt, M. C. 2007, ApJ, 658, 584

Lovis, C., et al. 2006, Nature, 441, 305

Mamajek, E. E., \& Hillenbrand, L. A. 2008, ApJ, 687, 1264

Marois, C., et al. 2008, Science, 322, 1348

Mayor, M., et al. 2004, A\&A, 415, 391

Metchev, S., Marois, C., \& Zuckerman, B. 2009, ApJ, 705, L204

Meyer, M. R., et al. 2008, ApJ, 673, L181

Moór, A., A., et al. 2006, ApJ, 644, 525

Moro-Martín, A., Wolf, S., \& Malhotra, R. 2005, ApJ, 621, 1079

Moro-Martín, A., Wyatt, M. C., Malhotra, R., \& Trilling, D. E. 2008, in The Solar System Beyond Neptune, ed. M. A. Barucci, H. Boehnhardt, D. P. Cruikshank, \& A. Morbidelli (Tucson, AZ: Univ. Arizona Press), 465

Moro-Martín, A., et al. 2007a, ApJ, 658, 1312

Moro-Martín, A., et al. 2007b, ApJ, 668, 1165
Moya, A., Amado, P. J., Barrado, D., García Hernández, A., Aberasturi, M., Montesinos, B., \& Aceituno, F. 2010, MNRAS, in press (arXiv:1003.3340) Müller, S., Löhne, T., \& Krivov, A. V. 2010, ApJ, 708, 1728

Nelson, R. P. 2003, MNRAS, 345, 233

Reidemeister, M., et al. 2009, A\&A, 503, 247

Saffe, C., Gómez, M., \& Chavero, C. 2005, A\&A, 443, 609

Saffe, C., Gómez, M., Pintado, O., \& González, E. 2008, A\&A, 490, 297

Sierchio, J. M., Rieke, G. H., Su, K. Y. L., Plavchan, P., Stauffer, J. R., \& Gorlova, N. I. 2010, ApJ, 712, 1421

Sousa, S. G., et al. 2008, A\&A, 487, 373

Stern, S. A. 1996, AJ, 112, 1203

Su, K. Y. L., et al. 2009, ApJ, 705, 314

Sylvester, R. J., Skinner, C. J., Barlow, M. J., \& Mannings, V. 1996, MNRAS, 279,915

Thébault, P., \& Augereau, J.-C. 2007, A\&A, 472, 169

Trilling, D. E., et al. 2008, ApJ, 674, 1086

Udry, S., et al. 2001, VizieR Online Data Catalog, 335, 60590

Valenti, J. A., \& Fischer, D. A. 2005, ApJS, 159, 141

Vogt, S. S., et al. 2005, ApJ, 632, 638

Weingartner, J. C., \& Draine, B. T. 2001, ApJ, 548, 296

Williams, J. P., \& Andrews, S. M. 2006, ApJ, 653, 1480

Wisdom, J. 1980, AJ, 85, 1122

Wolf, S., \& Hillenbrand, L. A. 2003, ApJ, 596, 603

Wright, J. T., Marcy, G. W., Butler, R. P., \& Vogt, S. S. 2004, ApJS, 152, 261 